\title{
THE NEXT GENERATION VIRGO CLUSTER SURVEY. XV. THE PHOTOMETRIC REDSHIFT ESTIMATION FOR BACKGROUND SOURCES
}

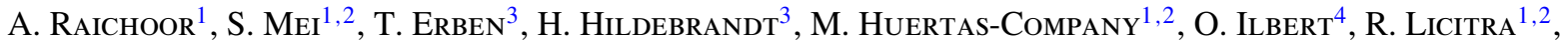 \\ N. M. Ball ${ }^{5,6}$, S. Boissier ${ }^{4,7}$, A. Bosellit ${ }^{4}$, Y.-T. Chen $^{8}$, P. Côté ${ }^{5}$, J.-C. Cuillandre ${ }^{9}$, P. A. Duc ${ }^{10}$, P. R. Durrell ${ }^{11}$, \\ L. Ferrarese ${ }^{5}$, P. Guhathakurta ${ }^{12}$, S. D. J. GwyN ${ }^{5}$, J. J. KavelaArs ${ }^{5}$, A. LançON ${ }^{13}$, C. Liu $^{14,15}$, L. A. MacArthur ${ }^{5}$, \\ M. Muller ${ }^{12}$, R. P. Muñoz ${ }^{13,16}$, E. W. Peng ${ }^{17,18}$, T. H. Puzia ${ }^{16}$, M. SAWicki ${ }^{19}$, E. Toloba ${ }^{12,20}$, \\ L. VAN WAERBEKE ${ }^{21}$, D. WoOds ${ }^{21}$, AND H. ZhANG ${ }^{17,18,22,23}$ \\ ${ }^{1}$ GEPI, Observatoire de Paris, CNRS, Université Paris Diderot, 61 Avenue de l'Observatoire, F-75014 Paris, France; anand.raichoor@obspm.fr \\ ${ }^{2}$ Université Paris Denis Diderot, F-75205 Paris Cedex 13, France \\ ${ }^{3}$ Argelander-Institut für Astronomie, University of Bonn, Auf dem Hügel 71, D-53121 Bonn, Germany \\ ${ }^{4}$ Aix Marseille Université, CNRS, Laboratoire d'Astrophysique de Marseille, UMR 7326, F-13388 Marseille, France \\ ${ }^{5}$ Herzberg Institute of Astrophysics, National Research Council of Canada, Victoria, BC V9E 2E7, Canada \\ ${ }^{6}$ Skytree, Inc., 1731 Technology Drive, Suite 700, San Jose, CA 95110, USA \\ ${ }^{7}$ INAF, Osservatorio Astronomico di Bologna, via Ranzani, 1, I-40127 Bologna, Italy \\ ${ }^{8}$ Insitute of Astronomy and Astrophysics, Academia Sinica, P.O. Box 23-141, Taipei 106, Taiwan \\ ${ }^{9}$ Canada-France-Hawaii Telescope Corporation, Kamuela, HI 96743, USA \\ ${ }^{10}$ Laboratoire AIM Paris-Saclay, CEA/IRFU/SAp, CNRS/INSU, Université Paris Diderot, F-91191 Gif-sur-Yvette Cedex, France \\ ${ }^{11}$ Department of Physics and Astronomy, Youngstown State University, Youngstown, OH 44555, USA \\ 12 UCO/Lick Observatory, Department of Astronomy and Astrophysics, University of California Santa Cruz, 1156 High Street, Santa Cruz, CA 95064, USA \\ ${ }_{13}$ Observatoire Astronomique de Strasbourg, Université de Strasbourg, CNRS, UMR 7550, 11 rue de l'Université, F-67000 Strasbourg, France \\ ${ }^{14}$ Center for Astronomy and Astrophysics, Department of Physics and Astronomy, Shanghai Jiao Tong University, 800 Dongchuan Road, Shanghai 200240, China \\ ${ }^{15}$ Shanghai Key Laboratory for Particle Physics and Cosmology, Shanghai Jiao Tong University, Shanghai 200240, China \\ ${ }^{16}$ Institute of Astrophysics, Pontificia Universidad Católica de Chile, Avenida Vicuña Mackenna 4860, 7820436 Macul, Santiago, Chile \\ ${ }^{17}$ Department of Astronomy, Peking University, Beijing 100871, China \\ ${ }^{18}$ Kavli Institute for Astronomy and Astrophysics, Peking University, Beijing 100871, China \\ ${ }^{19}$ Department of Astronomy and Physics, St. Mary's University, Halifax, NS B3H 3C3, Canada \\ ${ }^{20}$ Observatories of the Carnegie Institution for Science, 813 Santa Barbara Street, Pasadena, CA 91101, USA \\ ${ }^{21}$ Department of Physics and Astronomy, University of British Columbia, 6224 Agricultural Road, Vancouver, BC V6T 1Z1, Canada \\ ${ }^{22}$ Chinese Academy of Sciences South America Center for Astronomy, Camino El Observatorio 1515, Las Condes, Santiago, Chile \\ Received 2014 May 7; accepted 2014 October 8; published 2014 December 5
}

\begin{abstract}
The Next Generation Virgo Cluster Survey (NGVS) is an optical imaging survey covering 104 deg $^{2}$ centered on the Virgo cluster. Currently, the complete survey area has been observed in the $u^{*} g i z$ bands and one third in the $r$ band. We present the photometric redshift estimation for the NGVS background sources. After a dedicated data reduction, we perform accurate photometry, with special attention to precise color measurements through pointspread function homogenization. We then estimate the photometric redshifts with the Le Phare and BPZ codes. We add a new prior that extends to $i_{\mathrm{AB}}=12.5 \mathrm{mag}$. When using the $u^{*}$ griz bands, our photometric redshifts for $15.5 \mathrm{mag} \leqslant i \lesssim 23 \mathrm{mag}$ or $z_{\text {phot }} \lesssim 1$ galaxies have a bias $|\Delta z|<0.02$, less than $5 \%$ outliers, a scatter $\sigma_{\text {outl.rej. }}$, and an individual error on $z_{\text {phot }}$ that increases with magnitude (from 0.02 to 0.05 and from 0.03 to 0.10 , respectively). When using the $u^{*}$ giz bands over the same magnitude and redshift range, the lack of the $r$ band increases the uncertainties in the $0.3 \lesssim z_{\text {phot }} \lesssim 0.8$ range $\left(-0.05<\Delta z<-0.02, \sigma_{\text {outl.rej }} \sim 0.06,10 \%-15 \%\right.$ outliers, and $\left.z_{\text {phot.err. }} \sim 0.15\right)$. We also present a joint analysis of the photometric redshift accuracy as a function of redshift and magnitude. We assess the quality of our photometric redshifts by comparison to spectroscopic samples and by verifying that the angular auto- and cross-correlation function $w(\theta)$ of the entire NGVS photometric redshift sample across redshift bins is in agreement with the expectations.
\end{abstract}

Key words: galaxies: distances and redshifts - galaxies: high-redshift - galaxies: photometry techniques: photometric

Online-only material: color figures

\section{INTRODUCTION}

Many fields of astronomy have entered a new era with the advent of large surveys (e.g., the Sloan Digital Sky Survey, SDSS; York et al. 2000) that give access to homogeneous observations for a large number of objects (up to $10^{6}-10^{8}$ ). Such large surveys provide invaluable information for studies of galaxy evolution and cosmology based on homogeneous

\footnotetext{
${ }^{23}$ China-CONICYT Fellow.
}

measurements of a multitude of fundamental galaxy properties. In this context, a crucial quantity is the galaxy redshift.

Whereas spectroscopic redshifts (spec- $z$ 's) are unambiguous measurements, they are observationally too costly for $10^{6}-10^{8}$ objects. An alternative method, developed since the early 1960 s (e.g., Baum 1962; Koo 1985), is the use of photometric redshifts (photo- $z$ 's), which are estimated from photometry. Although less precise than spec- $z$ 's, photo- $z$ 's allow consistent measurement of redshifts for large numbers of galaxies, including relatively faint ones. The use of photo- $z$ 's for large surveys is widespread today (e.g., Ilbert et al. 2006, 2009; Coupon et al. 
2009; Bielby et al. 2012; Hildebrandt et al. 2012; Dahlen et al. 2013; Jouvel et al. 2014) and will be essential for future missions (e.g., EuCLID; Laureijs et al. 2011).

Existing codes to estimate photo-z's can be broadly classified into two categories: template fitting and empirical estimators. Template fitting codes (e.g., hyperz: Bolzonella et al. 2000; BPZ-Bayesian photometric redshift: Benítez 2000; Le Phare-Photometric Analysis for Redshift Estimate: Arnouts et al. 1999, 2002; Ilbert et al. 2006; EAZY-Easy and Accurate Redshifts from Yale: Brammer et al. 2008) use empirical or theoretical galaxy spectra to find, through fitting, the redshift/ template combination that best reproduces the observed colors, whereas empirical estimators (e.g., ANNz-Photometric redshifts using Artificial Neural Networks: Collister \& Lahav 2004; Ball et al. 2008; ArborZ: Gerdes et al. 2010) use a representative sample to train machines such as neural networks and reproduce the relation between the observed colors/magnitudes and the redshifts. The main limitations to estimate accurate photo- $z$ 's are the wavelength coverage of key spectral features (e.g., the Lyman break and the $4000 \AA /$ Balmer break) and the quality and homogeneity of the photometry. Hildebrandt et al. (2010) and Dahlen et al. (2013) have conducted thorough analyses on the performance of the most popular algorithms. Both studies agree that the majority of the codes provide quantitatively similar results. Dahlen et al. (2013) find that the photo$z$ 's accuracy depends strongly on the magnitude.

We present in this paper the estimation of photo- $z$ 's for the Next Generation Virgo Cluster Survey (NGVS; Ferrarese et al. 2012) with two template fitting codes: Le Phare and BPZ. The NGVS is a comprehensive optical imaging survey of the Virgo cluster from its core to its virial radius-covering a total area of $104 \mathrm{deg}^{2}$ - in the Canada-France-Hawaii Telescope (CFHT) $u^{*}$ griz bandpasses. ${ }^{24}$ The NGVS will serve as the optical reference survey over the Virgo cluster and will leverage the numerous other surveys targeting Virgo at shorter and longer wavelengths, such as, to cite only the most recent ones: the Galaxy Evolution Explorer Survey of Virgo in the ultraviolet (GUViCS; Boselli et al. 2011), the Next Generation Virgo Cluster Survey-Infrared in the near-infrared (NGVS-IR; Muñoz et al. 2014), the Herschel Virgo Cluster Survey in the far-infrared (HeViCS; Davies et al. 2010, 2012), or the Arecibo Legacy Fast ALFA Survey in the radio (ALFALFA; Giovanelli et al. 2005; Kent et al. 2008; Haynes et al. 2011).

The plan of this paper is as follows. Section 2 presents the data and their reduction. Section 3 details how we build the photometric catalogs. Section 4 presents the method to estimate the photo-z's, Section 5 analyzes their quality, and Section 6 gives a scientific validation of our photo- $z$ 's. We present our conclusions in Section 7.

In this paper, we adopt $H_{0}=70 \mathrm{~km} \mathrm{~s}^{-1} \mathrm{Mpc}^{-1}, \Omega_{m}=0.30$, and $\Omega_{\Lambda}=0.70$. All magnitudes are in the $\mathrm{AB}$ system and corrected for the Galactic foreground extinction using the Schlegel et al. (1998) maps.

\footnotetext{
24 The instrumental transmission curves can be found here: http://www1.cadc-ccda.hia-iha.nrc-cnrc.gc.ca/megapipe/docs/filters.html with the MegaCam instrument. We note that the NGVS observations have been performed with the new $i$-band filter ( $i$.MP9702, sometimes denoted $y$ ), which replaces the original $i$-band filter ( $i$.MP9701) that was damaged in 2008. Although we make the distinction in our pipeline, we write in this article $i$, regardless of the used passband, for simplicity.
}

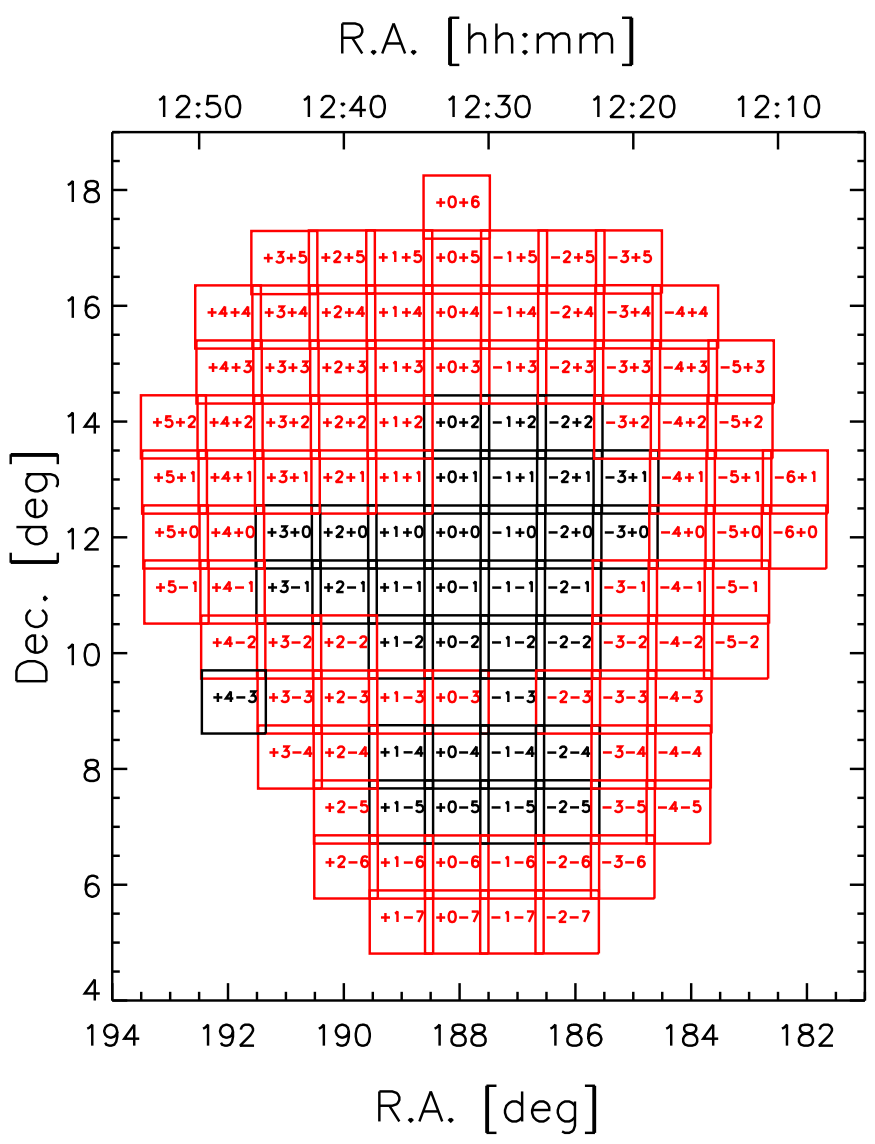

Figure 1. Layout of the NGVS survey footprint. Pointings in black (red, respectively) have coverage in $u^{*}$ griz ( $u^{*}$ giz, respectively).

(A color version of this figure is available in the online journal.)

\section{NGVSLenS DATA}

In this paper, we use a NGVS data set whose reduction is optimized for background science (see below): we label this data set NGVSLenS. We describe in this section the data acquisition along with their reduction.

\subsection{NGVSLenS Data}

The imaging data used in this article are from the NGVS (PI: L. Ferrarese). The goals of the survey, its implementation, and its observations have been described in detail in Ferrarese et al. (2012) and we only briefly repeat details relevant to this article.

The NGVS is a deep, $104 \mathrm{deg}^{2}$ multicolor optical imaging survey of the Virgo cluster. All the data are obtained with the MegaCam instrument ${ }^{25}$ (see Boulade et al. 2003), which is mounted on the CFHT. MegaCam is an optical multichip camera with a $9 \times 4$ CCD array $(2048 \times 4096$ pixels in each CCD; 0.'187 pixel scale; $\sim 1^{\circ} \times 1^{\circ}$ total field of view). NGVS observations were carried out with 117 discrete MegaCam pointings around the NGVS central position R.A. $=12^{\mathrm{h}} 32^{\mathrm{m}} 12^{\mathrm{s}}$, decl. $=12^{\mathrm{d}} 00^{\mathrm{m}} 19^{\mathrm{s}}$, which includes Virgo's $\mathrm{cD}$ M87. The exact NGVS survey layout is shown in Figure 1. In this article, we follow the NGVS convention to label individual NGVS pointings (see Figure 4 of Ferrarese et al. 2012) that indicate the approximate separation in degrees from the NGVS central position. For instance, pointing "NGVS-1+2" is about one degree west and two degrees north of the NGVS center.

\footnotetext{
25 http://www.cfht.hawaii.edu/Instruments/Imaging/Megacam/
} 
Table 1

Average Characteristics of the NGVS Coadded Data Used in This Study (See the Text for an Explanation of the Columns)

\begin{tabular}{lccc}
\hline \hline Filter & $\begin{array}{c}\text { Exposure Time } \\
(\mathrm{ks})\end{array}$ & $\begin{array}{c}m_{\mathrm{lim}}{ }^{\mathrm{a}} \\
(\mathrm{AB} \mathrm{mag})\end{array}$ & $\begin{array}{c}\text { Seeing } \\
\left({ }^{\prime \prime}\right)\end{array}$ \\
\hline$u^{*}(u . M P 9301)$ & 6.3 & $25.60 \pm 0.16$ & $0.83 \pm 0.07$ \\
$g($ g.MP9401) & 3.5 & $25.73 \pm 0.13$ & $0.77 \pm 0.08$ \\
$r($ r.MP9601) & 2.6 & $24.68 \pm 0.50^{\mathrm{b}}$ & $0.74 \pm 0.14$ \\
$i(i . M P 9702)$ & 2.3 & $24.41 \pm 0.13$ & $0.52 \pm 0.04$ \\
$z(z . M P 9801)$ & 4.6 & $23.62 \pm 0.16$ & $0.70 \pm 0.08$
\end{tabular}

Notes.

a $m_{\mathrm{lim}}$ is the $5 \sigma$ detection limit in a $2^{\prime \prime} 0$ aperture.

${ }^{\mathrm{b}}$ For the $r$ band, the minimum and maximum values for $m_{\mathrm{lim}}$ are 23.56 and 25.52, respectively.

We caution, however, that the data itself are processed with a slightly different convention ${ }^{26}$ (e.g., "NGVSm1p2"-read "NGVS minus 1 plus 2"-instead of "NGVS-1+2").

The complete NGVS area (117 pointings) is covered in four SDSS-like filters: $u^{*}$ (CFHT identification: u.MP9301), $g$ (g.MP9401), $i$ (i.MP9702), and $z$ (z.MP9801). Additionally, 34 pointings also benefit from $r$-(r.MP9601) band coverage (see Figure 1).

Table 1 contains observational details and provides averagequality characteristics of the final, coadded NGVS data used in this article. It lists the average observing time for the different filters, the mean limiting magnitudes, and the mean seeing values with their corresponding standard deviations over all 117 NGVS pointings. The seeing is estimated using the SEXTRACTOR (Bertin \& Arnouts 1996) parameter FWHM_IMAGE for stellar sources. Our limiting magnitude, $m_{\mathrm{lim}}$, is the $5 \sigma$ detection limit in a $2^{\prime \prime} .0$ aperture. ${ }^{27}$ The complete NGVS data set was obtained under very good observation conditions. In Figure 2 we show the full seeing distribution for all fields and filters (see also Figure 8 of Ferrarese et al. 2012). We specifically note the superb seeing distribution of the $i$ band: the complete survey was obtained in this filter with an exceptional seeing of $<0$ "' 6 .

As detailed in Ferrarese et al. (2012), the NGVS data are used for a large variety of science projects. The different applications can be split into three categories: (1) the foreground science, which will study the sources that are closer than the Virgo cluster; (2) the Virgo cluster itself; (3) and the background science, which uses the deep data to study the higher-redshift-background galaxy populations. As outlined in Ferrarese et al. (2012), the NGVS team performs different data processing and produces a large variety of data products optimized for different science applications. Foreground and Virgo science, requiring dedicated data processing, will be addressed in other publications by the NGVS collaboration (e.g., Durrell et al. 2014). The current study discusses the estimation of photo-z's of background sources, which are crucial for the background science. ${ }^{28}$ Future work will include the detection of high-redshift galaxy cluster candidates ( $R$. Licitra et al., in preparation) and strong and weak lensing studies (e.g., R. Gavazzi et al., in preparation). Photo-z studies

\footnotetext{
26 We avoid the "+/-" notation because of practical programming and processing reasons.

${ }_{27} m_{\text {lim }}=\mathrm{ZP}-2.5 \log \left(5 \sqrt{n_{\text {pix }}} \times \sigma_{\text {sky }}\right)$, where $\mathrm{ZP}$ is the magnitude zero-point, $n_{\text {pix }}$ is the number of pixels in a circle with radius $2 . \prime 0$, and $\sigma_{\text {sky }}$ is the sky-background noise variation.

28 Although they might also be useful for Virgo cluster science, for example, as in Boselli et al. (2011), where they permit background contamination removal.
}

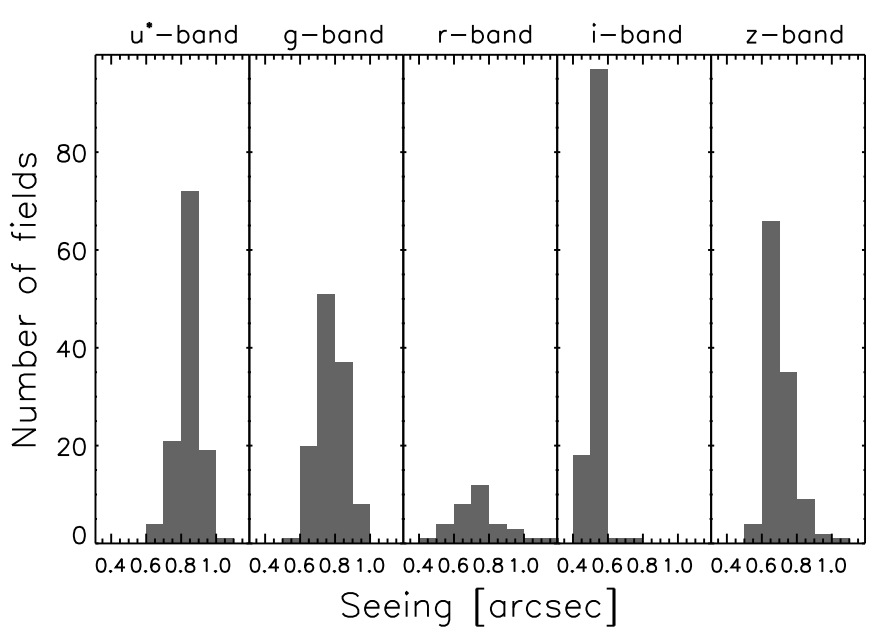

Figure 2. Seeing distributions for all coadded NGVS fields and filters.

of faint background sources require high-quality, deep, and carefully photometrically calibrated multicolor observations. In the following, we provide information on the preparation of the necessary data products.

\subsection{NGVSLenS Data Reduction}

To process the NGVS data for background science applications, we use the algorithms and processing pipelines (THELI) developed within the CFHTLS-Archive Research Survey (CARS; see Erben et al. 2009) and the Canada-France-Hawaii Telescope Lensing Survey (CFHTLenS; see Heymans et al. 2012; Hildebrandt et al. 2012; Erben et al. 2013, and http://cfhtlens.org). Both surveys originated from the Wide component of the Canada-France-Hawaii Telescope Legacy Survey (CFHTLS; Gwyn 2012), which was also obtained with MegaCam. In addition, the survey characteristics and the observational strategies of CFHTLS and NGVS are very similar. This allowed for a direct transfer of our CFHTLS expertise to NGVS. In the following, we only give a very short description of our procedures to arrive at the final coadded images for NGVS. All algorithms and prescriptions are described in detail in Erben et al. (2013). The interested reader should consult this article and the references therein. Below, we also give a more detailed analysis of the quality from our photometric calibration, which is crucial for the quality of photometric redshift estimates.

Our NGVS data processing consisted of the following steps.

1. Data sample. We start our data analysis with the ELIXIR ${ }^{29}$ preprocessed NGVS data available at the Canadian Astronomical Data Centre (CADC).$^{30}$ For the current study we used NGVS observations obtained from 2008 March 1 until 2013 June 12. The data were obtained under several CFHT programs (PI: L. Ferrarese: 08AC16, 09AP03, 09AP04, 09BP03, 09BP04, 10AP03, 10BP03, 11AP03, 11BP03, 12AP03, 12BP03, 13AC02, 13AP03; PI: Simona Mei: 08AF20; PI: Jean-Charles Cuillandre: 10AD99, 12AD99; and PI: Ying-Tung Chen: 10AT06). From the initial set, we reject all short exposed NGVS images. To be able to study bright cores of Virgo galaxies, the NGVS obtained, besides the primary scientific data, numerous short exposures in all pointings and filters (see Section 3.4 of Ferrarese et al. 2012). Because these exposures would not contribute

\footnotetext{
29 http://www.cfht.hawaii.edu/Instruments/Elixir/

30 http://www4.cadc-ccda.hia-iha.nrc-cnrc.gc.ca/cadc/
} 
an appreciable fraction to the total exposure time in each filter, we did not consider them further. We also do not use images whose observational conditions were marked as unfavorable by CFHT.

2. Single-exposure processing. The ELIXIR preprocessing includes a complete removal of the instrumental signature from the raw data (see also Magnier \& Cuillandre 2004). In addition, each exposure comes with all necessary photometric calibration information. Therefore, we only need to perform the following processing steps on single exposures: (1) we identify and mark individual exposure chips that should not be considered any further. This mainly concerns chips that are completely dominated by saturated pixels from a bright star. (2) We create sky-subtracted versions of the images. In the context of NGVS, we create a socalled local sky-background subtraction optimal for the study of faint background galaxies (see also Figure 11 of Ferrarese et al. 2012). In addition, we create a weight image for each science observation. This gives information on the relative noise properties of individual pixels and assigns a weight of zero to defective pixels (such as cosmic rays, hot and cold pixels, and areas of satellite tracks). (3) We use SEXTRACTOR to extract high signal-to-noise ratio sources $^{31}$ from the scientific image and weight information. These source catalogs are used to astrometrically and photometrically calibrate the data in the next processing step. In addition we perform an analysis of the point-spread function (PSF) anisotropy and use this information to reject images showing high stellar ellipticities. Highly elongated point sources are a good indication of tracking issues or other severe problems during an exposure.

3. Astrometric and photometric calibration. We use the SCAMP software $^{32}$ (see Bertin 2006) to astrometrically calibrate the NGVS survey. We use the Two Micron All Sky Survey (Skrutskie et al. 2006) as an astrometric reference and calibrate separately each filter from the NGVS patch (i.e., all fields) with SCAMP. Once an astrometric solution is established we use overlap sources from individual exposures to establish an internal, relative photometric solution for all exposures. We reject all exposures with an absorption of more than $0.2 \mathrm{mag}^{33}$ and rerun SCAMP on the remaining images. With the relative photometric solution and the ELIXIR zero-point information we estimate a patchwide photometric zero-point for each filter.

4. Image coaddition and mask creation. The next step of our image processing coadds the sky-subtracted exposures belonging to a pointing/filter combination with the SWARP program $^{34}$ (see Bertin et al. 2002). The stacking is performed with a statistically optimally weighted mean that takes into account sky-background noise, weight maps, and the SCAMP relative photometric zero-point information. As a final step we use the AUTOMASK tool (see Dietrich et al. 2007) to create image masks for all pointings. These masks flag bright, saturated stars and areas that would influence the analysis of faint background sources. For the NGVS, a reliable masking of bright Virgo members is particularly

\footnotetext{
31 We consider all sources having at least five pixels with at least $5 \sigma$ above the sky-background variation.

32 http://www.astromatic.net/software/scamp

33 More than $95 \%$ of the NGVS data has been obtained under at least good photometric conditions with an absorption of 0.05 mag or less. Our rejection limit of 0.2 mag turned out to be a very good conservative limit to reject the small fraction of images observed under poor photometric conditions.

34 http://www.astromatic.net/software/swarp
}

important for our purposes. The 117 generated masks have been visually checked (A.R.). In a typical NGVS pointing we lose about $20 \%-30 \%$ of the area because of masking, as can be seen in Figure 3, where we display those masks for a section of a field. In the figure we note that several algorithms and template masks are used to mask different artifacts, e.g., bright stars, short asteroid trails, or largescale bright objects. The artifacts we consider and the way we treat them are described in more detail in Erben et al. (2009).

5. Photometric calibration tied to the SDSS. The final step is specific to the NGVS, i.e., it has not been implemented in the released CFHTLenS. Taking advantage of the accurate internal photometric stability of the SDSS and its full coverage of the NGVS field, we tie our photometric calibration to the SDSS. We retrieve clean stars from the SDSS-DR10 (Ahn et al. 2014) and convert their Petrosian magnitude to the MegaCam photometric system using Equation (4) of Ferrarese et al. (2012). For each field and each filter available, we then measure the MAG_AUTO with SEXTRACTOR and correct for the offset between the two catalogs by taking the median value for a subset of bright nonsaturated stars $(\sim 500$ per field $)$. The typical uncertainty in this calibration step is of 0.05 mag in the griz bands and of $0.10 \mathrm{mag}$ in the $u^{*}$ band. We note that the THELI photometry is homogeneous over the NGVS field with a field-to-field standard deviation of $\sim 0.03 \mathrm{mag}$, as found in Erben et al. (2013).

\section{PHOTOMETRIC CATALOGS}

For simplicity, we hereafter are referring to NGVSLenS when referring to NGVS or the NGVSLenS data set and similarly to CFHTLenS with CFHTLS or the CFHTLenS data set.

In this section, we describe the procedure to build the photometric catalogs when the five $u^{*}$ griz bands are available (the procedure is similar when only the four $u^{*}$ giz bands are available). As studied in detail in Hildebrandt et al. (2012), a requirement to estimate precise photo- $z$ 's is accurate photometry, in particular, high-precision color measurements. To do so, we implement the following procedure (global mode of Hildebrandt et al. 2012): the $i$ band, which has the best seeing $\left(0{ }^{\prime \prime} .52 \pm 00^{\prime \prime} .04\right)$, is used to detect objects and estimate their total magnitude; then, for each field, all images are first homogenized to the same PSF and then used to estimate accurate colors.

\subsection{Global PSF Homogenization}

The photometric catalogs are constructed as described in Hildebrandt et al. (2012), who also describe the global PSF homogenization that is necessary to measure unbiased colors. According to the Hildebrandt et al. (2012) analysis done on the CFHTLenS data having properties similar to the NGVSLenS data (see Section 5 and Appendix B), the quality of the photo- $z$ 's obtained assuming a constant PSF across each field (global mode) provides satisfactory results, even when compared to that obtained when accounting for the PSF variations across each field (local mode). For this analysis, we hence consider that it is satisfactory to make the approximation that the PSF is constant over each field $\left(1 \mathrm{deg}^{2}\right)$ and can be described by a single Gaussian with width $\sigma_{\mathrm{PSF}}$.

For each field, we identify the band that has the largest seeing $\left(\sigma_{\mathrm{PSF}, \text { worst }}\right)$, and we bring the other four images to the same seeing by convolving them with a two-dimensional 

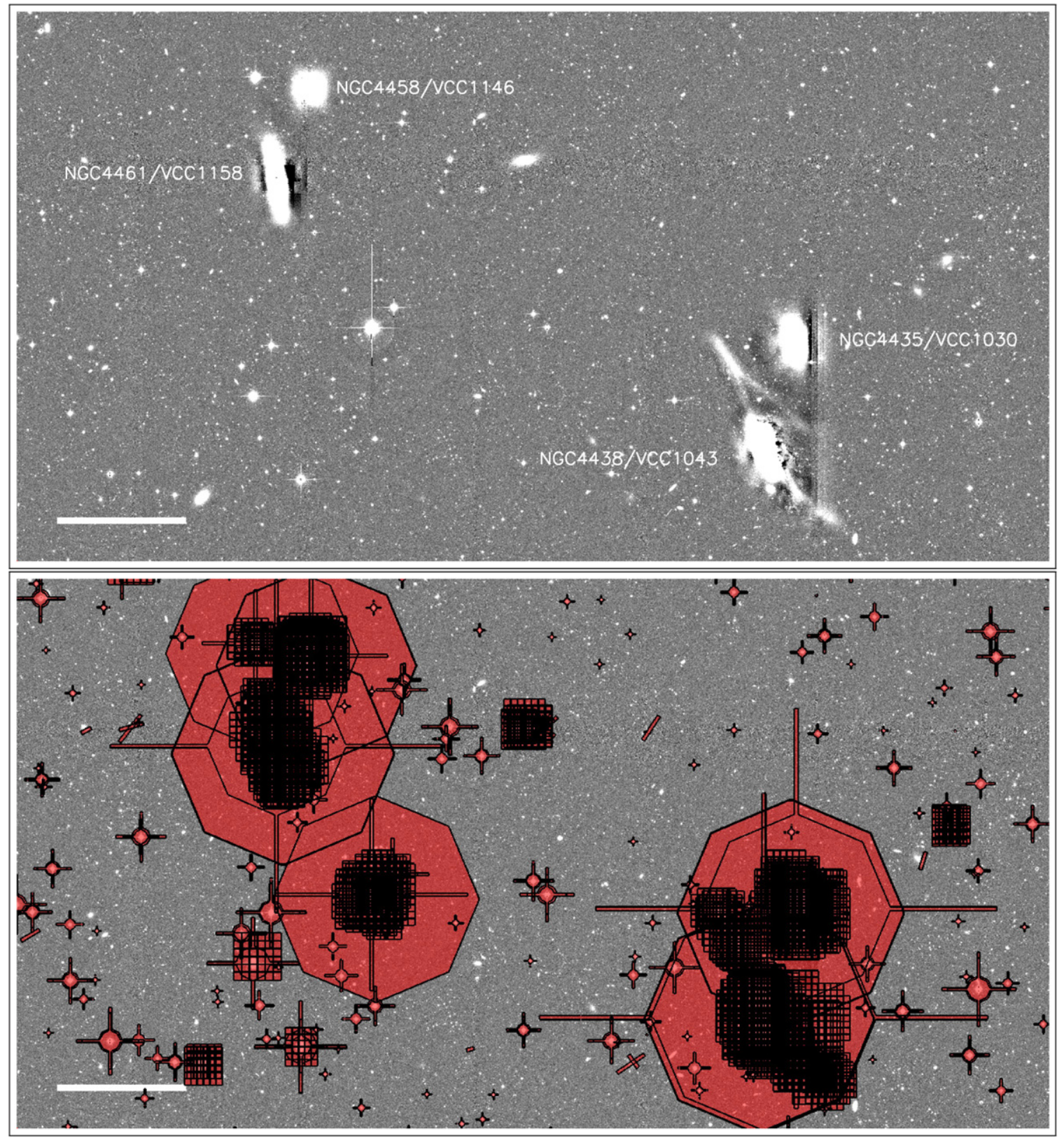

Figure 3. Image mask of a $40^{\prime} \times 25^{\prime}$. section of the NGVS-1+1 pointing. Our automatic masking reliably picks up bright stars and Virgo galaxies that would influence photometric analyses of faint background galaxies through their light halos (which are an artifact of the local background subtraction). Different mask shapes correspond to different artifacts (see text). We identify the prominent Virgo galaxies in this field. The ruler (white bar on the bottom left side) is $5^{\prime}$. wide.

(A color version of this figure is available in the online journal.)

Gaussian filter. For instance, if the $X$-band image has a PSF width $\sigma_{\mathrm{PSF}, \mathrm{X}}$, we convolve it with a Gaussian filter of width $\sqrt{\sigma_{\mathrm{PSF}, \text { worst }}^{2}-\sigma_{\mathrm{PSF}, X}^{2}}$. The 117 values of $\sigma_{\mathrm{PSF}, \text { worst }}$ have a mean value of $0.85 \pm 0.07$, and the band with $\sigma_{\mathrm{PSF}, \text { worst }}$ is the $u^{*}(g, r$, $i$, and $z$, respectively) band in 58 (45, 7, 0 , and 7 , respectively) fields.

\subsection{Photometry Method}

Multicolor catalogs in the $u^{*}$ griz bands are extracted from a set of these PSF homogenized images using SEXTRACTOR in dual-image mode, using the unconvolved $i$-band image as the detection image. One SEXTRACTOR run is performed on the unconvolved $i$ band image for detection, structural measurements, and estimation of the total $i$-band magnitudes (SExTRACTOR MAG_AUTO). Five SEXTRACTOR runs (dual-image mode, with the unconvolved $i$-band image as the detection image) are then performed with the PSF-matched $u^{*}$ griz images to measure accurate colors. Indeed, the obtained isophotal magnitudes MAG_ISO are measured on the same physical apertures, as we are using the same pixels (dual-mode) of PSF-matched images. From this procedure, we obtain accurate measurements for the colors and the $i$-band total magnitude; estimation of the total magnitude in the $u^{*} g r z$ bands can be obtained with (Hildebrandt et al. 2012)

$$
X_{\text {tot }}=i_{\text {tot }}+(X-i), \quad \text { with } X \in\left\{u^{*}, g, r, z\right\},
$$

where $X_{\text {tot }}$ and $i_{\text {tot }}$ are the total magnitudes in the $X$ and $i$ bands, respectively, and $(X-i)$ is the corresponding color index. 


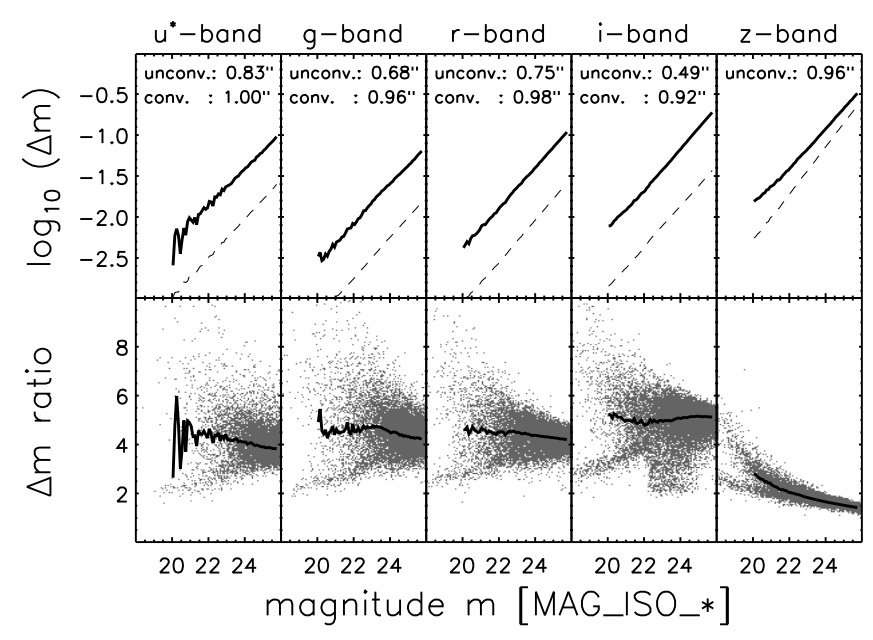

Figure 4. Re-estimated magnitude error $(\Delta m)$ properties for the NGVS $+0+0$ field. The continuous and dashed lines show the median values for 20 mag $\leqslant$ $m \leqslant 26$ mag. Top: we show $\Delta m$ as a function of magnitude $m$; our reestimated errors are shown as continuous lines, whereas SEXTRACTOR errors are denoted by dashed lines. We report for each band the seeing before and after convolution (for the NGVS+0+0 field, the $z$-band image is the one with the worst seeing, hence it is not convolved). Bottom: we show the ratio of our $\Delta m$ to SEXTRACTOR's $\Delta m$ as a function of magnitude $m$; points illustrate the individual distribution of objects (for clarity, we plot only one out of five points). SEXTRACTOR errors are on average underestimated by a factor of $\sim 4-5$ on convolved images and of $\sim 2$ on unconvolved images.

Note that we do not use the estimations of the total magnitude in the $u^{*} g r z$ bands. Additionally, we caution that these estimations will only be valid if the galaxies do not present strong color gradients.

\subsection{Photometric Errors}

In this study, we pay special attention to the photometric error estimation. Noise correlation introduced by image resampling during the reduction artificially decreases the pixel-to-pixel rms variations $\sigma_{1}$, which leads to an underestimation of the flux errors estimated by SExTRACTOR (e.g., Casertano et al. 2000). This effect is known, and the flux error underestimation factor for the CFHT/MegaCam optical bands is of the order of 1.5 (e.g., Ilbert et al. 2006; Coupon et al. 2009; Raichoor \& Andreon 2012). However, it can be much larger on convolved images, and the flux error underestimation factor can be of the order of five, as shown by our data analysis (see Figure 4). This phenomenon can be more pronounced in the presence of fringing (Ferrarese et al. 2012). We choose the following method to estimate the true background fluctuations, $\sigma_{\mathrm{bkg}}$ : regardless of whether the measurement is performed on a convolved or unconvolved image, we estimate $\sigma_{\mathrm{bkg}}$ in the unconvolved image, as it is not affected by the convolution process. ${ }^{35}$

For each of our 502 unconvolved images, we estimate $\sigma_{\mathrm{bkg}}$ by placing 2000 random apertures of a given size, which do not overlap with any detected objects (e.g., Labbé et al. 2003; Gawiser et al. 2006). We use circular apertures of area $n_{\text {pix }}$ centered at integer pixels, describe them by the linear size of the aperture defined as $N=\sqrt{n_{\text {pix }}}$, and fit a Gaussian to the histogram of aperture fluxes to yield $\sigma_{\text {bkg }}(N)$, the background fluctuation for a given aperture $N$. We apply this method for $0^{\prime \prime}<N<3^{\prime \prime}$. Then we fit the obtained $\sigma_{\mathrm{bkg}}(N)$ curve as a

\footnotetext{
35 Indeed, the $\sigma_{1}$ in the convolved image will have an artificially low value due to the noise correlation introduced by the convolution process.
}

Table 2

Median Values of the Image Noise Parameters Fitted with Equation (2)

\begin{tabular}{lccc}
\hline \hline Filter & $\begin{array}{c}\sigma_{1} \\
\left(\text { count s }^{-1}\right)\end{array}$ & $a$ & $b$ \\
\hline$u^{*}$ & $0.008 \pm 0.001$ & $0.995 \pm 0.037$ & $0.046 \pm 0.005$ \\
$g$ & $0.020 \pm 0.002$ & $1.029 \pm 0.038$ & $0.046 \pm 0.007$ \\
$r$ & $0.036 \pm 0.022$ & $1.054 \pm 0.047$ & $0.041 \pm 0.012$ \\
$y$ & $0.040 \pm 0.005$ & $1.025 \pm 0.039$ & $0.044 \pm 0.007$ \\
$z$ & $0.030 \pm 0.004$ & $0.865 \pm 0.059$ & $0.110 \pm 0.028$ \\
\hline
\end{tabular}

function of $N$, following Labbé et al. (2003) formalism:

$$
\sigma_{\mathrm{bkg}}(N)=\sigma_{1} \times\left(a N+b N^{2}\right)
$$

$\sigma_{1}=\sigma(1)$ are the pixel-to-pixel rms variations, measured through one pixel apertures for each unconvolved image for a given band and a given field. We present in Table 2 the median of the 502 fitted values for $\sigma_{1}, a$, and $b$.

Using error propagation and Poissonian uncertainties, the magnitude uncertainty $\Delta m$ for an object with a measured flux $F$ (in $\mathrm{ADU} \mathrm{s}{ }^{-1}$ ) and a pixel area $n_{\text {pix }}$ is obtained by accounting for the background noise and the Poissonian noise intrinsic to the object:

$$
\Delta m=\frac{2.5}{\ln 10} \times \frac{1}{w} \times \frac{\sqrt{F / g+\left[\sigma_{\mathrm{bkg}}\left(\sqrt{n_{\mathrm{pix}}}\right)\right]^{2}}}{F},
$$

where $g$ is the gain and $w$ is the square root of the number of single frames that contribute to the considered pixels divided by the number of single frames used to build the unconvolved image (which gives an estimation of the weight on the considered pixels; see Erben et al. 2013). We use the SExTRACTOR outputs ${ }^{36}$ for the flux $F$ and the pixel area $n_{\text {pix }}$ estimations.

We illustrate in Figure 4 how our estimated photometric uncertainties compare with those of SExTRACTOR for the NGVS+0+0 field. When the images are not convolved ( $z$ band for this field), we recover the usual SEXTRACTOR underestimation of $\sim 1.5$ because of pixel correlation. However, we see that the underestimation is much greater when SEXTRACTOR is run on a convolved image and that this underestimation is a function of the convolution kernel and of the object's magnitude (and size). When plotting the individual objects (bottom panels), we remark that stars have a different behavior because of their small $n_{\text {pix }}$ compared to galaxies of similar magnitude.

\section{PHOTOMETRIC REDSHIFT ESTIMATION}

With the photometric catalogs described in the previous section in hand, we are able to estimate the photo- $z$ 's. In this section we describe the procedure used to estimate them. In Table 3, we summarize the setup parameters used in this analysis.

\subsection{Code: Le Phare and BPZ}

In the present study, we use two template-based codes to estimate photo-z's: Le Phare ${ }^{37}$ (Arnouts et al. 1999, 2002; Ilbert et al. 2006) and BPZ (Benítez 2000; Benítez et al. 2004;

\footnotetext{
36 For AUTO (isophotal, respectively) magnitudes, the flux $F$ is given by FLUX_AUTO (FLUX_ISO, respectively), and the area $n_{\text {pix }}$ is given by $\pi \times A_{-}$IMAGE $\times$B_IMAGE $\times$KRON_RADIUS ${ }^{2}$ (ISOAREAF_IMAGE, respectively).

37 http://www.cfht.hawaii.edu/ arnouts/LEPHARE/lephare.html
} 
Table 3

Photo- $z$ Setup Parameters Summary

\begin{tabular}{ll}
\hline \hline Parameter & \multicolumn{1}{c}{ Comment } \\
\hline Template set & El, Sbc, Scd, Im, SB2, SB3 (Capak et al. 2004) \\
Prior & Appendix A (Le Phare prior for $i>20$ mag, extended down to $i=12.5$ mag) \\
$E(B-V)$ reddening & Le Phare: $0 \leqslant E(B-V) \leqslant 0.25 ;$ BPZ: none \\
Reddening law & Le Phare: Prevot et al. (1984); BPZ: none \\
Minimum photometric error & rgiz band: 0.05 mag; $u^{*}$ band: 0.10 mag
\end{tabular}

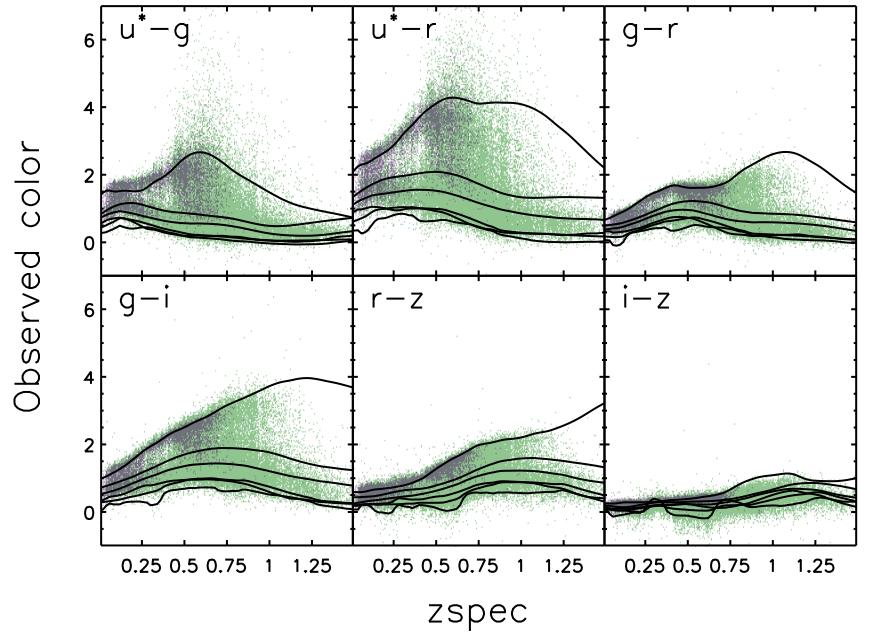

Figure 5. Observed colors as a function of $z_{\mathrm{spec}}$ : magenta and green dots show our NGVSLenS and CFHTLenS spectroscopic samples, respectively (both described in Section 5.1); black lines represent the colors predicted by our adopted template set. Our template set satisfactorily reproduces the observed colors.

(A color version of this figure is available in the online journal.)

Coe et al. 2006). In addition to having been widely used and tested, Hildebrandt et al. (2010) and Dahlen et al. (2013) have shown that these two codes provide satisfactory results.

\subsection{Templates}

For both codes, we use the recalibrated template set of Capak et al. (2004), which is built from the four Coleman et al. (1980) observed galaxy spectra (El, Sbc, Scd, and Im), with two additional observed starburst templates from Kinney et al. (1996). We note that, when running Le Phare and BPZ, those six templates are linearly interpolated into $\sim 60$ templates so as to have a better sampling of the color space.

A requirement of our template set is its ability to reproduce the observed colors. In Figure 5, we display the observed colors for our spectroscopic sample ( $\sim 83,000$ galaxies; described in Section 5.1), along with the colors predicted by the templates. Our templates cover the observed colors in a satisfactory way. We note that galaxies having a $u^{*}-r$ color redder than the models are a minority: for instance, less than $3 \%$ of galaxies with $0.4<z_{\text {spec }}<0.9$ have $u^{*}-r>5$ mag.

Le Phare offers the possibility to include galaxy internal reddening, $E(B-V)$, as a free parameter during the fit. When running Le Phare for spectral types later than $\mathrm{Sbc}$, we let $E(B-V)$ act as a free parameter $(0 \leqslant E(B-V) \leqslant 0.25)$ using the Small Magellanic Cloud extinction law (Prevot et al. 1984; see Coupon et al. 2009). We have tested that our results of Section 5 are independent of the use of another extinction law (Large Magellanic Cloud; Fitzpatrick 1986) or a larger range of possible reddening $(0 \leqslant E(B-V) \leqslant 0.5)$.

\subsection{Fitting Procedure and New Prior}

Overall, both codes run in a similar way using a Bayesian approach. This approach, and more precisely the use of a prior, allows the estimate of robust photo- $z$ 's; its advantage over the maximum-likelihood method is described in detail in Benítez (2000). Briefly, in the maximum-likelihood method, a priori assumptions are implicitly made on the choice of the explored parameter space. The Bayesian approach, with the use of a prior, implements a priori knowledge in a more systematic and detailed way. Regarding the photo- $z$ estimation, thanks to the existence of large, intensive spectroscopic surveys coupled with imaging (e.g., SDSS; zCOSMOS: Lilly et al. 2007; DEEP2: Cooper et al. 2008; and VVDS: Le Fèvre et al. 2013), we have some precise and statistically robust knowledge about the relationship between spec- $z$, magnitude, and spectral type. This a priori knowledge is used to favor more physical solutions and brings essential constraints when there are few bandpasses to estimate the photo- $z$ 's, as is the case in our work.

We now succinctly present the Bayesian approach (please refer to Benítez 2000, for a detailed presentation). For a given galaxy with an observed magnitude $m_{0}$ in a reference band ( $i$ band here) and observed colors $C$, the posterior $p\left(z \mid m_{0}, C\right)$, i.e., the probability for this galaxy to be at redshift $z$ given the observed data, can be expressed as a sum of probabilities over the basis formed by the different spectral distribution types, $T$, belonging to our template set (see Section 4.2 and Table 3):

$$
p\left(z \mid m_{0}, C\right)=\sum_{T} p\left(z, T \mid m_{0}, C\right),
$$

where $p\left(z, T \mid m_{0}, C\right)$ is the probability of the galaxy redshift being $z$ and the galactic spectral template type being $T$. According to Bayes' theorem, $p\left(z, T \mid m_{0}, C\right)$ is proportional to the product of the likelihood $p(C \mid T, z)$ of observing those colors for a galaxy of spectral template type $T$ at redshift $z$ and of the prior $p\left(z, T \mid m_{0}\right)$, which translates the a priori probability for a galaxy of magnitude $m_{0}$ to be at redshift $z$ and have a spectral template type $T$ :

$$
p\left(z, T \mid m_{0}, C\right) \propto p(C \mid T, z) \times p\left(z, T \mid m_{0}\right) .
$$

To account for zero-point uncertainty (see Section 2.2), we add in quadrature an uncertainty of $0.05 \mathrm{mag}$ in the griz bands and of $0.10 \mathrm{mag}$ in the $u^{*}$ band. Those account for the typical uncertainties in our photometric calibration explained in Section 2.2. Both codes provide the redshift posterior distribution. We take as the photo- $z$ estimate the median of this posterior distribution. We choose to use the median of the $p(z)$ because it improves the redshift estimation in the most difficult cases in which the algorithm cannot define a clear peak of the distribution (see also the Dahlen et al. 2013 analysis when comparing results from different photo- $z$ algorithms). This corresponds to the Z_ML output of $L e$ 
Phare; BPZ does not provide this output: we compute it based on the output posterior. Regarding the photo- $z$ uncertainties, we use the boundary of the interval including $68 \%$ of the redshift probability distribution function. Le Phare outputs those as Z_ML68_LOW and Z_ML68_HIGH; BPZ provides only $95 \%$ uncertainty on $z_{\text {phot }}$ : for each object, we compute, based on the output posterior, the $68 \%$ confidence interval as defined in Le Phare.

\subsubsection{Introduction of a New Prior}

Le Phare and BPZ were designed for high-redshift studies: both codes use similar priors for $i>20$ mag galaxies, built with observed data. However, the priors used for $i<20$ mag galaxies are not calibrated on observed data and, as a consequence, do not provide satisfying constraints. A direct outcome is lowquality photo- $z$ 's for $z_{\text {spec }} \lesssim 0.2$ objects, for which the photo- $z$ 's have either a large scatter with Le Phare (e.g., see Figure 11 of the CFHTLS/Wide T0007 paper $^{38}$ ) or are biased toward high values with BPZ (e.g., see Figure 4 of Erben et al. 2009). As a result of the large area covered by the NGVSLenS, $i<20$ mag galaxies represent a nonnegligible fraction of our sample: Hildebrandt et al. (2012) already noticed this issue with the CFHTLenS data - the BPZ prior biasing the posterior against low photo- $z$ 's - and implemented an ad hoc solution. We tackle this issue in a more systematic way by extending the prior to bright objects. We use the SDSS Galaxy Main Sample spectroscopic survey (York et al. 2000; Strauss et al. 2002; Ahn et al. 2014) to establish the prior for $12.5 \mathrm{mag}<i \leqslant 17$ mag galaxies and extrapolate the prior for $17 \mathrm{mag}<i \leqslant 20 \mathrm{mag}$ galaxies. The construction of this extended prior is detailed in Appendix A.

\subsubsection{No Photometric Recalibration}

When using the template-fitting method, it is common to add some photometric offsets during the fitting (e.g., Brodwin et al. 2006; Ilbert et al. 2006; Hildebrandt et al. 2010; Dahlen et al. 2013) because they can improve the accuracy of the estimated photo- $z$ 's. They may correct for various effects, such as imprecise photometric calibration, mismatch between the used templates and spectral energy distributions of observed galaxies, imprecise filter throughputs, or different properties of source images when using multicolor catalogs. These offsets are calculated with an iterative process comparing the colors predicted from the templates with the colors measured for a spectroscopic subsample.

We have tested the computation of such offsets (using the bright objects of our spectroscopic sample described in Section 5.1). We find small offsets $(<0.03$ mag in absolute value) and hence do not use them in the present study. Our approach is in agreement with the analysis of Hildebrandt et al. (2012), who concluded that using PSF-matched photometry decreases the offset amplitude.

\section{PHOTOMETRIC REDSHIFT ACCURACY}

In this section we present an analysis of our photo- $z$ 's to quantify their accuracy.

As detailed below, our spectroscopic sample over the NGVSLenS field is rather shallow $(z \lesssim 0.8)$ and highly biased at $z \gtrsim 0.3$. In order to assess the quality of our photometric redshifts up to $z<1.5$, we use the CFHTLenS data, which are covered by deep and intensive spectroscopic surveys.

\footnotetext{
38 ftp://ftpix.iap.fr/pub/CFHTLS-zphot-T0007/cfhtls_wide_T007_v1.2_ Oct2012.pdf
}

Those CFHTLenS data have been imaged with the same telescope, instrument, and filters (except for the $i$-band filter, which was replaced), have similar depth, and have been reduced with the same THELI pipeline. Starting from the CFHTLenS THELI coadded images, we reestimate for the CFHTLenS the photometry and photo-z's with the THELI pipeline, including our modifications described in the previous sections (including the photometric calibration tied to the SDSS; see (v) of Section 2.2). We confirm a posteriori the close similarity of the CFHTLenS and NGVSLenS data sets in Appendix B.

We can thus compare our photo-z's with two complementary spectroscopic samples over the NGVSLenS and the CFHTLenS fields, as described in the next section.

In this analysis, we exclude very low redshift $(z \leqslant 0.01)$ objects, mainly Virgo objects, as those are either spectroscopically confirmed (Virgo galaxies) or have redshifts difficult to constrain with optical data only (Virgo globular clusters-GCs-and U1tracompact dwarf galaxies-UCDs). Muñoz et al. (2014) show that near-infrared data are crucial to diagnose those populations. Those objects, along with Galactic stars, are excluded either using the spec- $z$ for the spectroscopic samples, or using the criteria presented in Appendix $\mathrm{C}$ for the photometric samples. We remark that our pipeline computes a photo- $z$ for those objects, but we do not analyze it here.

In Section 5.1, we present the samples that we use to analyze our photo-z's. We quantify, as a function of magnitude or redshift, the accuracy of our photo-z's when they are estimated with the $u^{*}$ griz bands (Sections 5.2 and 5.3) or with the $u^{*} g i z$ bands (Section 5.4). Section 5.5 presents a joint analysis of photo- $z$ dependence on magnitude and redshift.

\subsection{Samples Definition}

In this section, we present and define the samples used to analyze the accuracy of our photo-z's. We first present the two spectroscopic samples covering the NGVSLenS and the CFHTLenS. We then present the photometric samples covering the NGVSLenS. The properties of those samples are summarized in Table 4.

\subsubsection{Spectroscopic Sample over the NGVSLenS}

The NGVSLenS field is covered by several spectroscopic surveys having different target selections. The entire NGVSLenS field is covered by the SDSS, providing $\sim 23,500$ galaxy spec-z's: $\sim 40 \%$ come from the Galaxy Main Sample, which is magnitude-limited ( $r \leqslant 17.77$; Strauss et al. 2002), and have $\left\langle z_{\text {spec }}\right\rangle=0.11 \pm 0.05$; the remaining $\sim 60 \%$ come from different target selection functions, mainly targeting luminous red galaxies (LRGs; Eisenstein et al. 2001; Dawson et al. 2013), have $\left\langle z_{\text {spec }}\right\rangle=0.48 \pm 0.13$, and represent by selection the most luminous and reddest galaxies at each redshift (e.g., see Figure 5 and the bottom panel of Figure 6).

Other spectroscopic programs targeting candidate GCs or UCDs (MMT, PI: E. Peng; E. Peng et al., in preparation; AAT, PI: P. Côté: Zhang et al., 2014; H. Zhang et al., in preparation) provide us with $\sim 2500$ spec- $z$ 's $\left(\left\langle z_{\text {spec }}\right\rangle=0.16 \pm 0.12\right)$. We also gathered $\sim 90$ spec- $z$ 's from the Virgo Dwarf Globular Cluster Survey taken with the Keck telescope (Keck, PI: P. Guhathakurta; P. Guhathakurta et al., in preparation): those are faint $(i=23.2 \pm 1.2 \mathrm{mag}$ ) emission line galaxies that were either purposely targeted (e.g., missclassified as GC, dwarf elliptical) or are serendipitous sources that happened to land on the "blank sky" portions of the slits. Because of its faintness, this subsample is very different from the rest of our NGVSLenS 

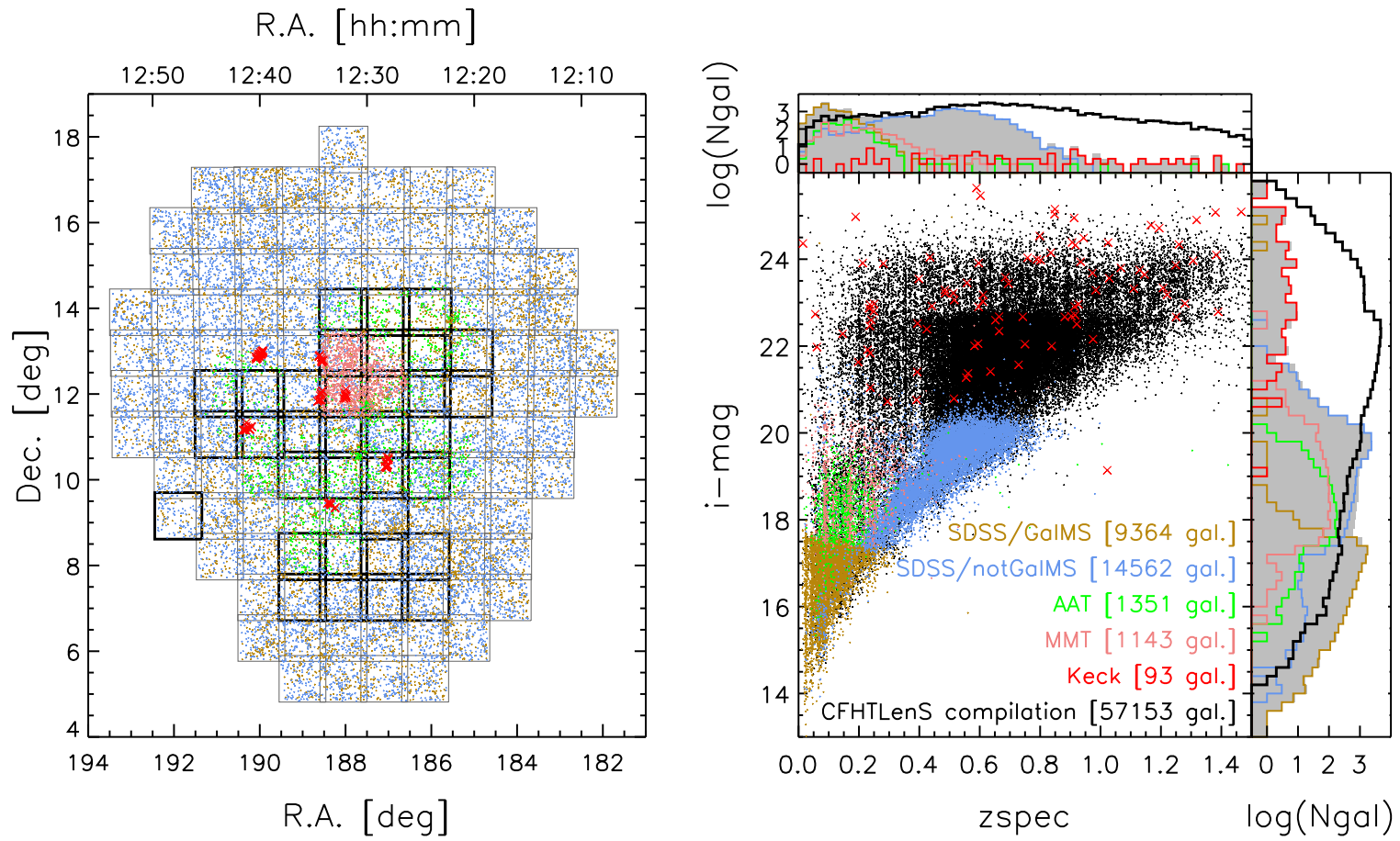

Figure 6. Spectroscopic sample properties. Spec-z's from the SDSS/Galaxy Main Sample (SDSS/other programs, AAT, MMT, and Keck, respectively) survey are in gold points (blue points, green points, coral points, and red crosses, respectively) symbols. The black points are the CFHTLenS spectroscopic sample (SDSS; VVDS F02/F22; DEEP2/EGS; and VIPERS W1/W4). Left: we show the spatial distribution of the NGVSLenS spectroscopic sample; the fields covered by the $u^{*} g r i z$ bands have thick black outlines. Right: we show the $i$-band magnitude as a function of $z_{\text {spec }}$; the filled gray histograms represent the whole NGVSLenS spectroscopic sample. (A color version of this figure is available in the online journal.)

Table 4

Properties of the Samples Used in Section 5

\begin{tabular}{|c|c|c|c|c|c|c|}
\hline Survey & $\begin{array}{l}\text { Number of Galaxies } \\
\qquad\left(10^{3}\right)\end{array}$ & $\begin{array}{c}\text { Area } \\
\left(\mathrm{deg}^{2}\right)\end{array}$ & Band Coverage & $\left\langle z_{\text {spec }}\right\rangle^{\mathrm{a}}$ & $\begin{array}{c}\langle i\rangle^{\mathrm{a}} \\
(\mathrm{mag})\end{array}$ & Reference \\
\hline \multicolumn{7}{|c|}{ Spectroscopic sample over the NGVSLenS field $\left(0.01 \leqslant z_{\text {spec }}<1.5\right)$} \\
\hline SDSS/GalMS & 9.2 & 104 & $u^{*}$ griz/ $/ u^{*} g i z$ & $0.11 \pm 0.05$ & $16.8 \pm 1.1$ & (1) \\
\hline SDSS/notGalMS & 14.3 & 104 & $u^{*}$ griz/u*giz & $0.48 \pm 0.13$ & $19.3 \pm 0.8$ & (2) \\
\hline AAT & 1.4 & $\sim 30$ & $u^{*}$ griz $/ u^{*}$ giz & $0.15 \pm 0.09$ & $18.2 \pm 0.6$ & (3) \\
\hline MMT & 1.1 & $\sim 4$ & $u^{*}$ griz & $0.19 \pm 0.10$ & $18.7 \pm 0.8$ & (4) \\
\hline Keck & 0.1 & $\ldots$ & $u^{*} \operatorname{griz} / u^{*} \mathrm{giz}$ & $0.72 \pm 0.37$ & $23.2 \pm 1.2$ & (5) \\
\hline Compiled & 26.1 & 104 & $u^{*}$ griz/ $/ u^{*}$ giz & $0.32 \pm 0.21$ & $18.3 \pm 1.5$ & $\ldots$ \\
\hline \multicolumn{7}{|c|}{ Spectroscopic sample over the CFHTLenS field $\left(0.01 \leqslant z_{\text {spec }}<1.5\right)$} \\
\hline SDSS/GalMS & 0.8 & $\sim 24$ & $u^{*}$ griz & $0.11 \pm 0.05$ & $16.6 \pm 0.6$ & (1) \\
\hline SDSS/notGalMS & 5.6 & $\sim 42$ & $u^{*}$ griz & $0.41 \pm 0.21$ & $19.0 \pm 1.6$ & (2) \\
\hline VVDS/F22 & 4.0 & $\sim 3$ & $u^{*}$ griz & $0.52 \pm 0.23$ & $21.4 \pm 0.9$ & (6) \\
\hline VVDS/F02 & 5.0 & $<1$ & $u^{*}$ griz & $0.69 \pm 0.30$ & $22.7 \pm 1.2$ & (6) \\
\hline DEEP2/EGS & 12.1 & $<1$ & $u^{*}$ griz & $0.71 \pm 0.32$ & $22.5 \pm 1.2$ & (7) \\
\hline VIPERS & 29.6 & $\sim 17$ & $u^{*}$ griz & $0.68 \pm 0.16$ & $21.7 \pm 0.8$ & (8) \\
\hline Compiled & 57.2 & 42 & $u^{*}$ griz & $0.65 \pm 0.25$ & $21.6 \pm 1.5$ & $\ldots$ \\
\hline \multicolumn{7}{|c|}{ Photometric samples over the NGVSLenS field } \\
\hline NGVSLenS/phot23 & 576.7 & $\sim 30$ & $u^{*}$ griz & & $21.9 \pm 1.0$ & This paper \\
\hline NGVSLenS/phot 24 & $1,263.5$ & $\sim 30$ & $u^{*}$ griz & $\cdots$ & $22.8 \pm 1.1$ & This paper \\
\hline
\end{tabular}

Notes.

${ }^{a}$ Mean and standard deviation.

References. (1) Strauss et al. 2002; (2) Eisenstein et al. 2001, Dawson et al. 2013; (3) Zhang et al. (2014); H. Zhang et al. (in preparation); (4) E. W. Peng et al. (in preparation); (5) P. Guhathakurta et al. (in preparation); (6) Le Fèvre et al. 2005, 2013; (7) Davis et al. 2003, Newman et al. 2013; (8) Guzzo et al. 2013. 


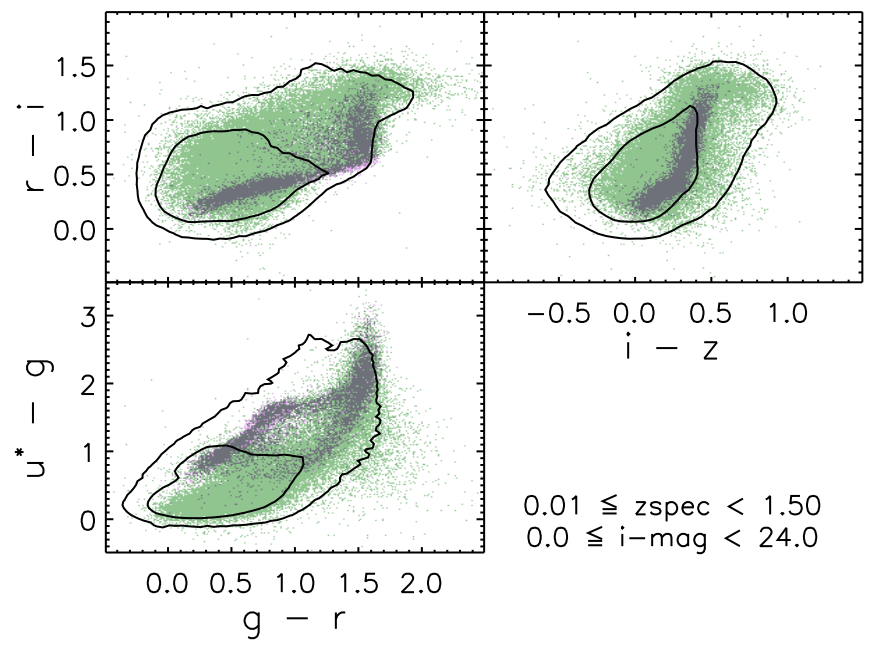

Figure 7. Coverage of the color-color space by our spectroscopic samples. Magenta and green dots show our NGVSLenS and CFHTLenS spectroscopic samples, respectively. Contours represent the $68 \%$ and $95 \%$ loci of our NGVSLenS/ phot 24 photometric sample. Our spectroscopic samples satisfactorily cover the colors of the photometric sample.

(A color version of this figure is available in the online journal.)

spectroscopic subsamples and provides a unique opportunity to probe-even sparsely-our photo- $z$ 's up to $z_{\mathrm{spec}} \sim 1.5$. for $21 \mathrm{mag}<i<24.5 \mathrm{mag}$.

We display in Figure 6 the spatial distribution (top) and $i$-band magnitude versus $z_{\text {spec }}$ distribution (bottom) of our spec- $z$ compilation. Our spec- $z$ compilation over the NGVSLenS is very heterogeneous and rather shallow in redshift $\left(\sim 50 \%\right.$ with $\left.z_{\text {spec }} \lesssim 0.3\right)$. At low redshift $\left(z_{\text {spec }} \lesssim 0.3\right)$, it covers a wide range of colors and galaxy types; however, at higher redshifts $\left(z_{\text {spec }} \gtrsim 0.3\right)$, it is severely biased toward LRGs.

\subsubsection{Spectroscopic Sample over the CFHTLenS}

To complement this spectroscopic sample, we use the public CFHTLenS data (Erben et al. 2013) covering three intensive and deep spectroscopic surveys: the DEEP2 Galaxy Redshift Survey over the Extended Groth Strip (DEEP2/EGS; Davis et al. 2003; Newman et al. 2013), the VIMOS Public Extragalactic Redshift Survey (VIPERS; Guzzo et al. 2013), and the F02 and F22 fields of the VIMOS VLT Deep Survey (VVDS; Le Fèvre et al. 2005, 2013). The DEEP2/EGS survey is a magnitude-limited survey ( $R \leqslant 24.1 \mathrm{mag}, \sim 12,000$ galaxies), as is the VVDS (17.5 mag $\leqslant i \leqslant 24$ mag for the F02 field, $~ 5000$ galaxies; $i \leqslant 22.5 \mathrm{mag}$ for the F22 field, $\sim 4000$ galaxies), whereas the VIPERS ( $\sim 30,000$ galaxies) is preselected for color and mainly targets objects in the range $0.5 \lesssim z_{\text {spec }} \lesssim 1.2$ down to $i \sim 22.5 \mathrm{mag}$. For these three surveys, we select only galaxies having a secure redshift (flag $=\{3,4\}$ for the DEEP2/EGS and VVDS; $3 \leqslant$ flag $<5$ for the VIPERS). Furthermore, the CFHTLenS fields covering these three deep spectroscopic surveys are also covered by the SDSS, which provides additional spec- $z$ 's ( $\sim 6400$ galaxies, mostly obtained from surveys other than the Galaxy Main Sample). In Figure 6, we present our CFHTLenS spectroscopic sample as black points. This includes $\sim 57,000$ spec- $z$ 's and spreads over $\sim 42 \mathrm{deg}^{2}$, which mitigates the field-to-field variations in exposure times.

\subsubsection{Photometric Sample over the NGVSLenS}

To see how the properties of our spectroscopic samples compare to the NGVSLenS photometric data-and to which

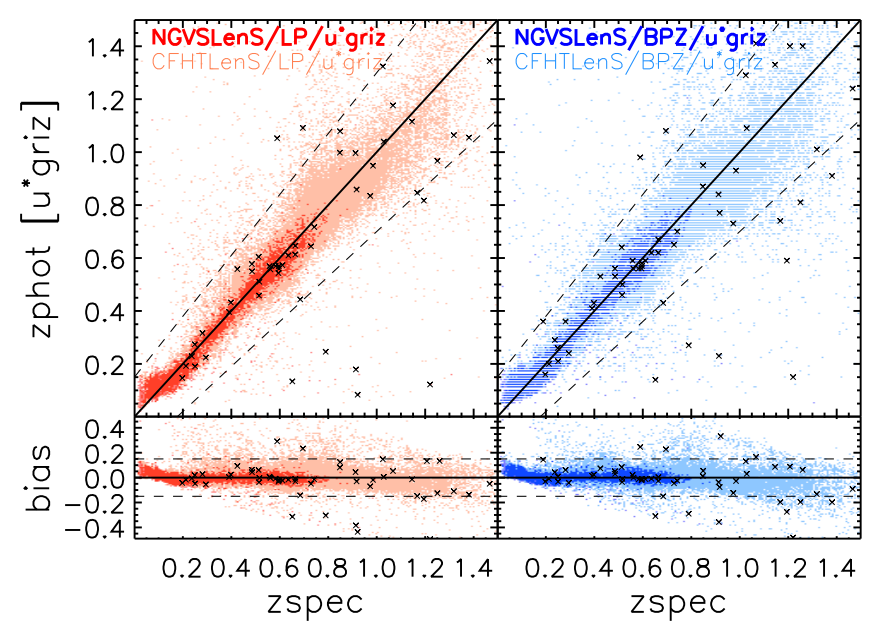

Figure 8. Photo-z's with $u^{*}$ griz, bands with Le Phare (left, red) and BPZ (right, blue). Dark thick symbols represent the NGVSLenS spectroscopic sample (low redshift); light thin symbols represent the CFHTLenS spectroscopic sample (high redshift). We highlight with black crosses the NGVSLenS Keck subsample.

(A color version of this figure is available in the online journal.)

extent they are representative thereof-we define two photometric samples as follows. We select objects: (1) lying in the 34 NGVSLenS fields having $u^{*}$ griz band coverage (we exclude the overlap regions), (2) with valid photometry in those five bands, and (3) not classified as a star or GC using the criteria described in Appendix C.

We define NGVSLenS/phot23 (NGVSLenS/phot24, respectively) as the corresponding NGVSLenS photometric sample, when further applying a $i<23 \mathrm{mag}(i<24 \mathrm{mag}$, respectively) cut, which comprises $5.8 \times 10^{5}\left(12.6 \times 10^{5}\right.$, respectively) objects.

In Figure 7, we show how our combined spectroscopic sample (over the NGVSLenS and the CFHTLenS) overlaps with the color-color space of the NGVSLenS data (NGVSLenS/phot24 sample). The colored dots represent our spectroscopic sample, and the contours are the $68 \%$ and $95 \%$ loci of the observed NGVSLenS photometric objects. We remark that the "blue" sides (i.e., toward the bottom left side) of the $95 \%$ contours that are not well covered by our spectroscopic sample are mainly populated by 23 mag $<i<24$ mag objects; in other words, our spectroscopic sample spans with high coverage the color-color space for $i<23$ mag objects and satisfactorily covers the 23 mag $<i<24$ mag objects (the regions within the $68 \%$ contours are well-populated by our spectroscopic sample).

\subsection{Comparison with Spec-z's}

In this section we analyze how our estimated photo- $z$ 's compare with our spectroscopic sample. We use the full spectroscopic sample (NGVSLenS and CFHTLenS) in the redshift range $0.01 \leqslant z_{\text {spec }}<1.5$, without any selection in magnitude. For each object in our spectroscopic sample, we calculate $\Delta z=\left(z_{\text {phot }}-z_{\text {spec }}\right) /\left(1+z_{\text {spec }}\right)$ and classify it as an outlier if $|\Delta z|>0.15$. For each considered sample, we report bias: the median value of $\Delta z ;$ outl.: the percentage of outliers; and $\sigma_{\text {outl.rej. }}$ : the standard deviation of $\Delta z$ when outliers have been excluded. These quantities are used to facilitate comparison with other works; as mentioned in Hildebrandt et al. (2012), the outlier definition is arbitrary.

We present in Figure 8 how our photo- $z$ 's compare with spec- $z$ 's for our two spectroscopic samples and for both codes, 


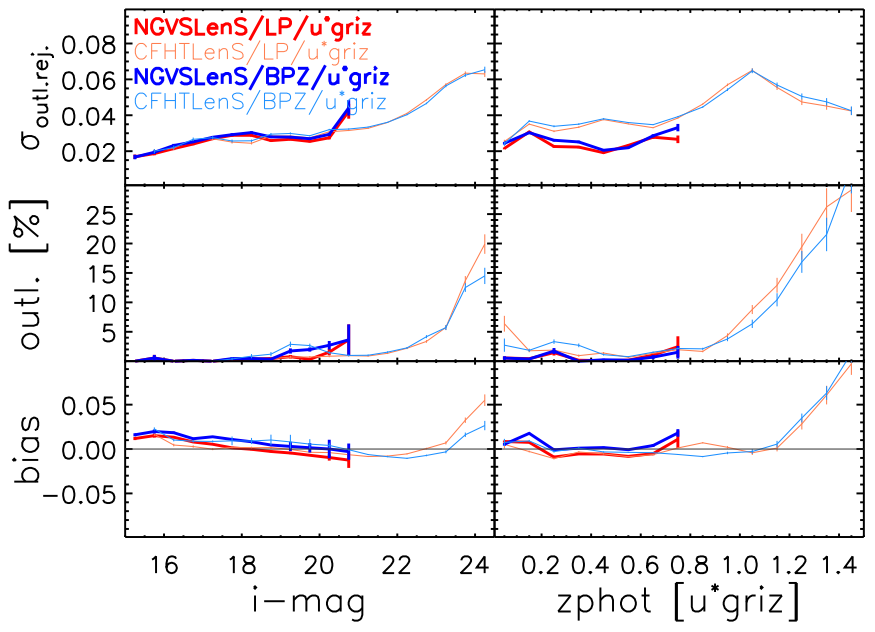

Figure 9. Statistics for photo-z's (estimated with $u^{*}$ griz bands) as a function of magnitude (left) and redshift (right). Photo- $z$ 's estimated with Le Phare are in red, and those estimated with BPZ are in blue. Dark thick lines represent the NGVSLenS spectroscopic sample (low redshift), and light thin lines represent the CFHTLenS spectroscopic sample (high redshift). We report quantities only for the bins where we have more than 50 galaxies. Error bars are calculated assuming a Poissonian distribution. Our photo- $z$ 's estimated with the $u^{*} \mathrm{griz}$ bands are more accurate for $i \lesssim 23$ mag or $z_{\text {phot }} \lesssim 1$.

(A color version of this figure is available in the online journal.)

Le Phare (left, red) and BPZ (right, blue). The NGVSLenS objects (low redshift) are in dark thick symbols, and the CFHTLenS objects (high redshift) are in light thin symbols.

At first sight, we see that both codes provide satisfactory photo- $z$ 's over the range $0.1 \lesssim z_{\text {spec }} \lesssim 1$ and that the overall behavior of our spectroscopic samples over the NGVSLenS and the CFHTLenS fields is consistent in the overlap regions. We notice that although statistically small, the NGVSLenS Keck subsample has photo- $z$ 's in broad agreement with the other NGVSLenS subsamples and with the CFHTLenS spectroscopic sample, strengthening our choice of using the CFHTLenS data at high redshift.

We show in Figure 9 a quantitative analysis of how the three quantities, bias, $\sigma_{\text {outl.rej., and outl., depend on the measured }}$ magnitude and the estimated photo- $z$.

First, we observe that the two codes (BPZ and Le Phare) and the two data sets (NGVSLenS and CFHTLenS) provide consistent behavior over our tested ranges in magnitude or photo- $z$. This observation a posteriori validates our assumption, namely, that the NGVSLenS and CFHTLenS data have very similar properties. However, when looking at the $\sigma_{\text {outl.rej. }}$ as a function of photo- $z$, there is a clear difference between the NGVSLenS and CFHTLenS samples, which arises from the nature of the two spectroscopic samples in a given range. In the $0.3 \lesssim z_{\text {phot }} \lesssim 0.6$ range, the NGVSLenS sample has a significantly smaller $\sigma_{\text {outl.rej. }}$. This is a direct consequence of our NGVSLenS spectroscopic sample in this redshift range being highly biased toward LRGs. These galaxies have on average brighter magnitudes and smaller photometric errors (e.g., better defined $4000 \AA$ break than the average galaxy), thus making the photo- $z$ estimation easier. For instance, the typical value of the $i$ band magnitude at redshift $\sim 0.5$ is 19.5 mag for our NGVSLenS spectroscopic sample versus $21.5 \mathrm{mag}$ for our CFHTLenS spectroscopic sample (see right panel of Figure 6): a direct consequence is that the prior for those LRGs is significantly more peaked and at lower redshifts, thus constraining the posterior more. Indeed, as illustrated in Figure 10, the [0.11,

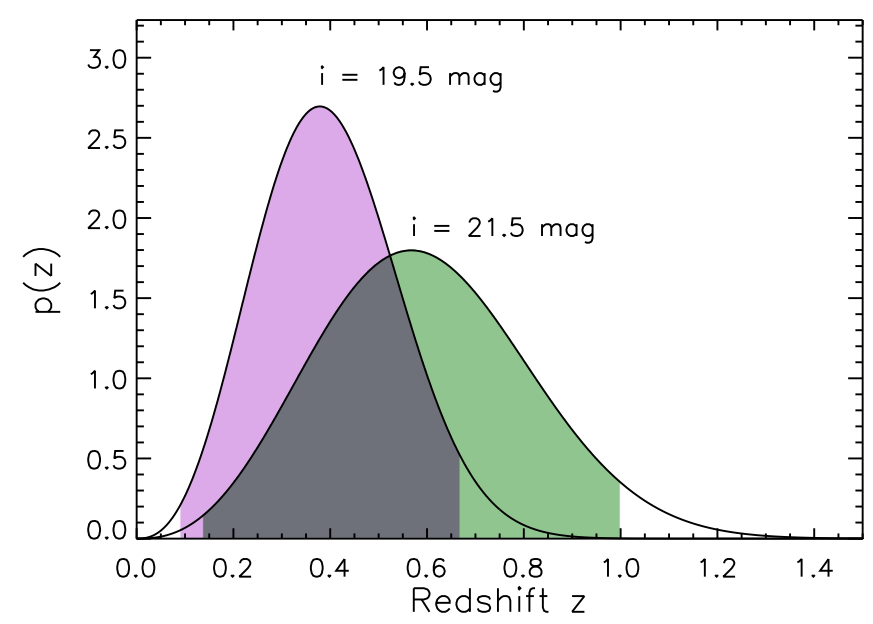

Figure 10. Example of the prior for an elliptical template for two magnitudes representative of our spectroscopic samples at a redshift of $z \sim 0.5: i=$ 19.5 mag (in magenta, representative of our NGVSLenS spectroscopic sample) and $i=21.5 \mathrm{mag}$ (in green, representative of our CFHTLenS spectroscopic sample). The shaded areas enclose $95 \%$ of the prior. As a consequence, brighter galaxies will have on average sharper posteriors.

(A color version of this figure is available in the online journal.)

0.65 ] redshift interval includes $95 \%$ of the prior for an elliptical galaxy with $i=19.5 \mathrm{mag}$, whereas for an elliptical galaxy with $i=21.5$ mag the corresponding redshift interval is $[0.14,1.00]$.

Another trend that illustrates this point is the outlier rate at $z_{\text {phot }} \lesssim 0.2$ : the CFHTLenS sample has $3 \%-5 \%$ outliers against $<1 \%$ for the NGVSLenS sample. Again, this can be explained by the characteristic $i$-band magnitude in this redshift range: when considering objects with $z_{\text {phot }}<0.2$, only $5 \%$ of the NGVSLenS sample has $i>20$ mag against $27 \%$ for the CFHTLenS sample. The fainter galaxies of the CFHTLenS will have a much broader prior; hence the photo- $z$ will be less constrained.

The quality of our photo- $z$ 's decreases with increasing magnitude or redshift. For instance, with both codes, the bias becomes significant $(>0.02)$ for faint $(i \gtrsim 23 \mathrm{mag})$ or high- $z(z \gtrsim 1.2)$ objects, and the $\sigma_{\text {outl.rej. }}$ goes from $\sim 0.02$ for bright/low- $z$ objects to $\sim 0.06$ for faint/high- $z$ objects. For $z_{\text {phot }} \gtrsim 1.2$, our optical data do not bracket the $4000 \AA$ break, and the photo- $z$ 's are less reliable.

The quality of our photo- $z$ for $i \gtrsim 17.5 \mathrm{mag}$ is consistent with that from the CFHTLenS data computed with Le Phare (Ilbert et al. 2006; Coupon et al. 2009) or with BPZ (Hildebrandt et al. 2012; Erben et al. 2013). However, we obtain more robust photo- $z$ 's down to at least $i \simeq 15.5 \mathrm{mag}$ because of the new prior that we introduce for the brightest galaxies.

\subsection{Individual Photo-z Uncertainty $z_{\text {phot,err. }}$}

In this section, we discuss the individual photo- $z$ uncertainty estimation, which we label $z_{\text {phot,err. }}$ For Le Phare, we use Z_ML68_LOW and Z_ML68_HIGH, which represent for each galaxy the boundary of the interval including $68 \%$ of the redshift probability distribution function; BPZ provides only $95 \%$ uncertainty on $z_{\text {phot }}$ : for each object, we compute, based on the output posterior, the $68 \%$ confidence interval as defined in Le Phare.

In the top panels of Figure 11, we present the percentage of objects having $z_{\text {spec }}$ within $z_{\text {phot }} \pm z_{\text {phot,err. }}$ for our spectroscopic sample and as a function of measured magnitude or photo- $z$. 


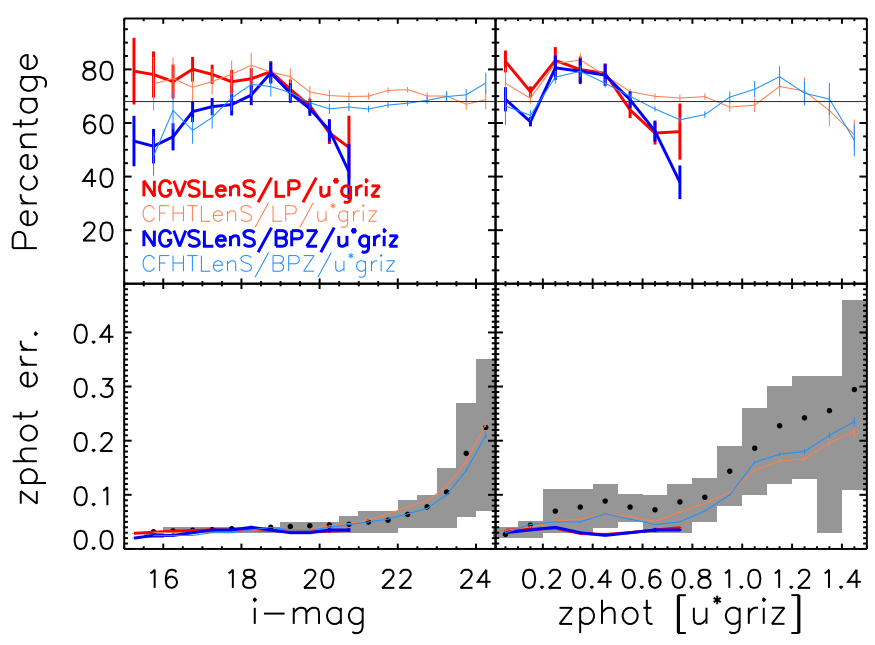

Figure 11. Properties of, $z$ phot,err., the individual error estimation on the photo- $z$ (estimated with $u^{*}$ griz bands) as a function of magnitude (left) and redshift (right). Photo-z's estimated with Le Phare are in red, and those estimated with BPZ are in blue. Dark thick lines represent the NGVSLenS spectroscopic sample (low redshift), and light thin lines represent the CFHTLenS spectroscopic sample (high redshift). Error bars are calculated assuming a Poissonian distribution. Top: percentage of objects having $z_{\text {spec }}$ within $z_{\text {phot }} \pm z_{\text {phot,err. }}$. The fact that these percentages are close to $68 \%$, as expected from the $z_{\text {phot,err. }}$ uncertainty estimation, means that our estimated $z$ phot,err. are realistic. Bottom:

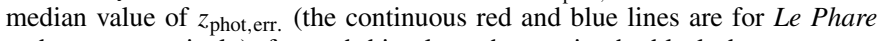
and BPZ, respectively); for each bin along the $x$ axis, the black dots represent

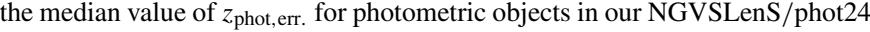
sample, and the gray shaded areas represent the regions enclosing $68 \%$ of their distribution. Our spectroscopic sample is representative of the photometric sample.

(A color version of this figure is available in the online journal.)

On average, this percentage is close to $68 \%$, which means that our estimated individual $z_{\text {phot,err. }}$ are realistic.

We see a departure from this behavior for the NGVSLenS sample at $i \gtrsim 20 \mathrm{mag}$ and at $z_{\text {phot }} \gtrsim 0.5$ : this is again due to the fact that our NGVSLenS spectroscopic sample in this magnitude/redshift range is highly biased toward LRGs. Indeed, as those objects are bright with a clear $4000 \AA$ break, the estimated individual $z_{\text {phot,err. }}$ is small; hence the $z_{\text {spec }}$ can be outside of the $z_{\text {phot }} \pm z_{\text {phot,err. }}$ interval. This means that the uncertainties obtained for the photometric redshift are underestimated for LRGs: for this population, setting a minimal

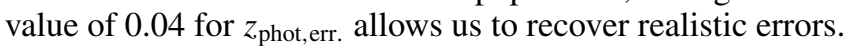

In the lower panels of Figure 11, we present how the median value of $z_{\text {phot,err. }}$ varies as a function of the measured magnitude and $z_{\text {phot }}$. The median value of $z_{\text {phot,err. depends strongly on }}$ magnitude. The gray area shows the region including the $68 \%$ of our NGVSLenS/phot24 sample photometric sample. For the spectroscopic sample, the median value of $z_{\text {phot,err. }}$ is comparable to the scatter $\sigma_{\text {outl.,rej. }} \times\left(1+z_{\text {spec }}\right)$ for $i<23$ mag or $z_{\text {phot }}<1$ (i.e., when the factor $\left(1+z_{\text {spec }}\right)$ is taken into account). Our spectroscopic sample is representative of the overall behavior of all photometric objects, even if the spectroscopic sample has lower median uncertainties as a function of redshift because the majority of the photometric sample includes objects fainter than the spectroscopic sample $(i>22 \mathrm{mag})$.

\subsection{Photo-z's without the $r$ Band}

As mentioned in Section 2, a majority (83/117 fields) of the NGVSLenS field has not been imaged yet with the $r$ band. In this section, we present the quality of our photo- $z$ 's when the $r$ band

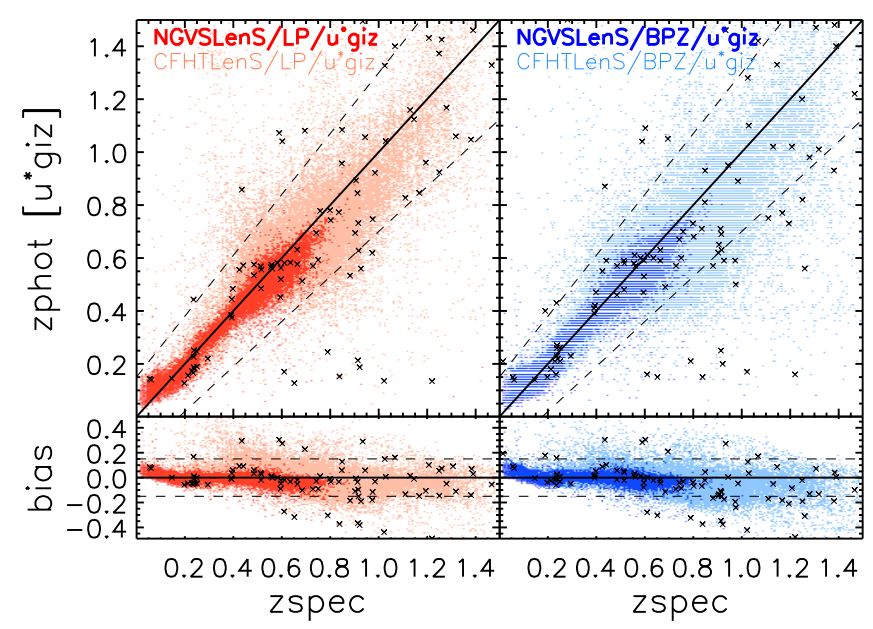

Figure 12. Photo-z's with $u^{*}$ giz bands with Le Phare (left, red) and BPZ (right, blue). Dark thick symbols represent the NGVSLenS spectroscopic sample (low redshift), and light thin symbols represent the CFHTLenS spectroscopic sample (high redshift). We highlight with black crosses the NGVSLenS Keck subsample.

(A color version of this figure is available in the online journal.)

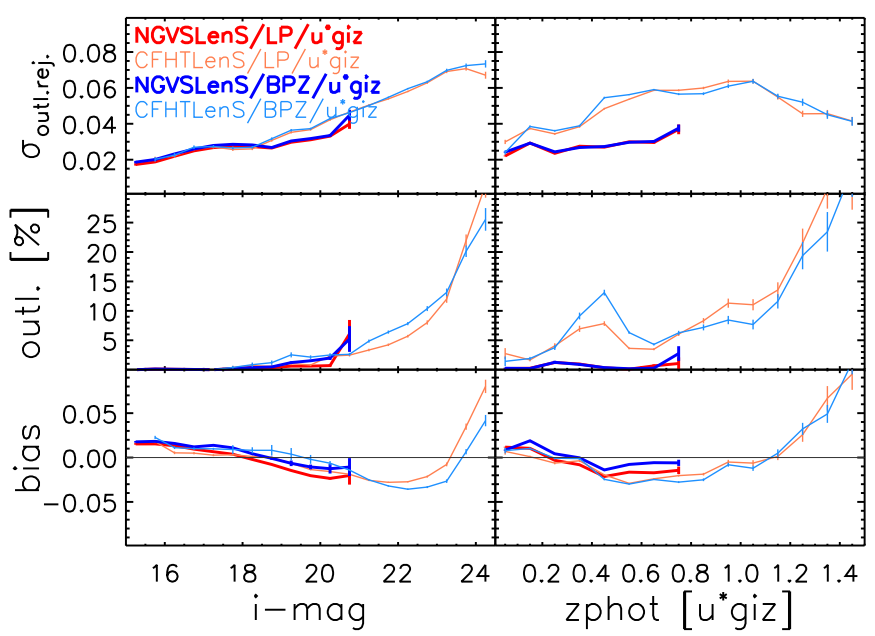

Figure 13. Statistics for photo- $z$ 's (estimated with $u^{*} g i z$ bands) as a function of magnitude (left) and redshift (right). Photo-z's estimated with Le Phare are in red, and those estimated with BPZ are in blue. Dark thick lines represent the NGVSLenS spectroscopic sample (low redshift), and light thin lines represent the CFHTLenS spectroscopic sample (high redshift). We report quantities only for the bins where we have more than 50 galaxies. Error bars are calculated assuming a Poissonian distribution. When the $r$ band is missing, the quality of our photo- $z$ 's decreases for the three plotted quantities in the $0.3 \lesssim z_{\text {phot }} \lesssim 0.8$ range and in the $i \gtrsim 21$ mag range (bias, outl., and $\sigma_{\text {outl.rej. can increase more }}$ than $100 \%$ ).

(A color version of this figure is available in the online journal.)

is not available. To estimate it, we recalculated the photo- $z$ 's using only the $u^{*}$ giz bandpasses for the 34 NGVSLenS fields having $r$ band coverage and for the CFHTLenS fields, thus using our full spectroscopic sample ( $\sim 83,000$ galaxies).

Figures 12 and 13 summarize the properties of the photo$z$ 's for our spectroscopic sample when the $r$ band is missing. We also present in Figure 14 the comparison of the statistics for the photo- $z$ 's estimated with or without the $r$ band for our CFHTLenS spectroscopic sample. In our CFHTLenS spectroscopic sample, our photo- $z$ 's are more scattered in the $0.3 \lesssim z_{\text {spec }} \lesssim 0.8$ range, where the $r$ band filter is essential to constrain the $4000 \AA$ break. This effect is less pronounced for 

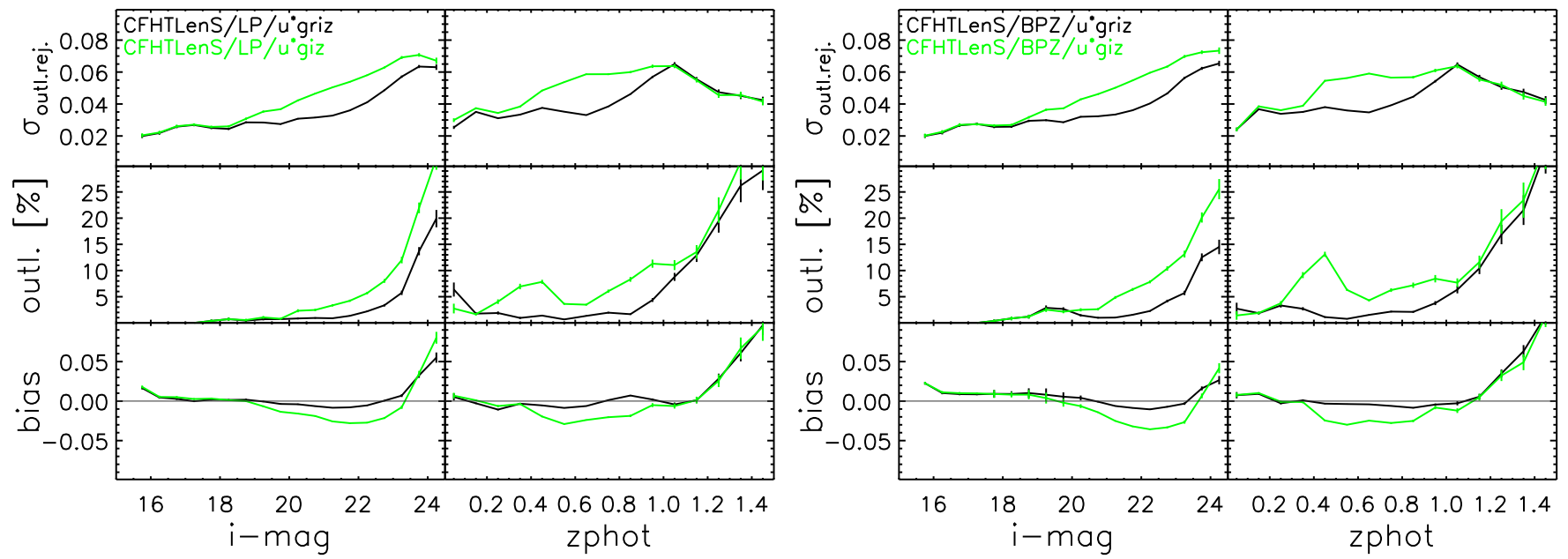

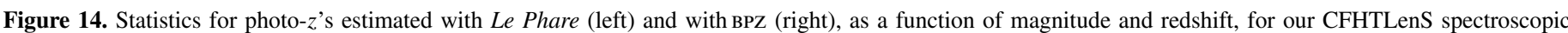

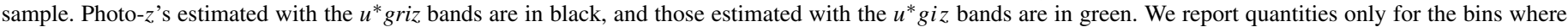

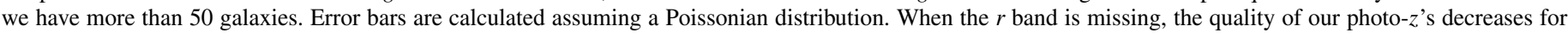
the three plotted quantities in the $0.3 \lesssim z_{\text {phot }} \lesssim 0.8$ range and in the $i \gtrsim 21$ mag range (bias, outl., and $\sigma_{\text {outl.rej. }}$ can increase more than $100 \%$ ).

(A color version of this figure is available in the online journal.)

our NGVSLenS spectroscopic sample because, as discussed in Section 5.2 (see also Figure 10), our NGVSLenS spectroscopic sample is highly biased toward LRGs (i.e., thus not representative of the general galaxy population) in this redshift range: the prior-more peaked and at lower redshift than for average galaxies at similar redshift-helps to obtain fewer false values for the posterior. When we compare the statistics (e.g., Figure 14), when the $r$ band is missing, the outliers rate increases significantly in the $0.3 \lesssim z_{\text {phot }} \lesssim 0.8$ range and peaks at $\sim 10 \%-15 \%$. We remark that the overall behavior of the two codes is similar.

The observation that the photo- $z$ quality decreases in the $0.3 \lesssim z_{\text {spec }} \lesssim 0.8$ range is supported only by our CFHTLenS spectroscopic sample. Using the results of Section 5.2, we can assume that our photo- $z$ 's estimated with five bands $u^{*}$ griz are unbiased down to $i<23 \mathrm{mag}$. Under this assumption, we can use our NGVSLenS/phot23 sample (see Section 5.1 and Table 4), i.e., the whole NGVSLenS photometric sample with $i<23$ mag and covered by the five bands, to probe how photometric redshifts change if the $r$ band is missing.

Figure 15 compares the photo- $z$ 's estimated with $u^{*} \mathrm{giz}$ bands versus those estimated with the $u^{*}$ griz bands for the two codes and by magnitude bins. We consider here objects from our NGVSLenS/phot23 photometric sample, thus $5.8 \times 10^{5}$ objects. This confirms the result obtained with the CFHTLenS spectroscopic sample, which is that a significant number of objects with $0.3 \lesssim z_{\text {phot }} \lesssim 0.8$ are outliers.

Under the same assumption-our photo-z's estimated with the $u^{*}$ griz bands are globally unbiased down to $i<$ $23 \mathrm{mag}$ - we can produce a plot similar to Figure 13, but using our NGVSLenS/phot23 photometric sample, as above. In Figure 16, we present the statistics for our photo-z's estimated with the $u^{*} g i z$ bands, using the photo- $z$ 's estimated with the $u^{*}$ griz bands as a proxy of $z_{\text {spec }}$. Most of the features of Figure 13 are rather accurately reproduced with this sample of $5.8 \times 10^{5}$ photometric objects. These statistics are dominated by faint galaxies, less biased by the LRGs, and are consistent with statistics obtained with the CFHTLenS spectroscopic sample.

Figure 16 strengthens the results shown in Figure 13, as the statistics are closely reproduced by using a sample of $5.8 \times 10^{5}$ photometric objects.

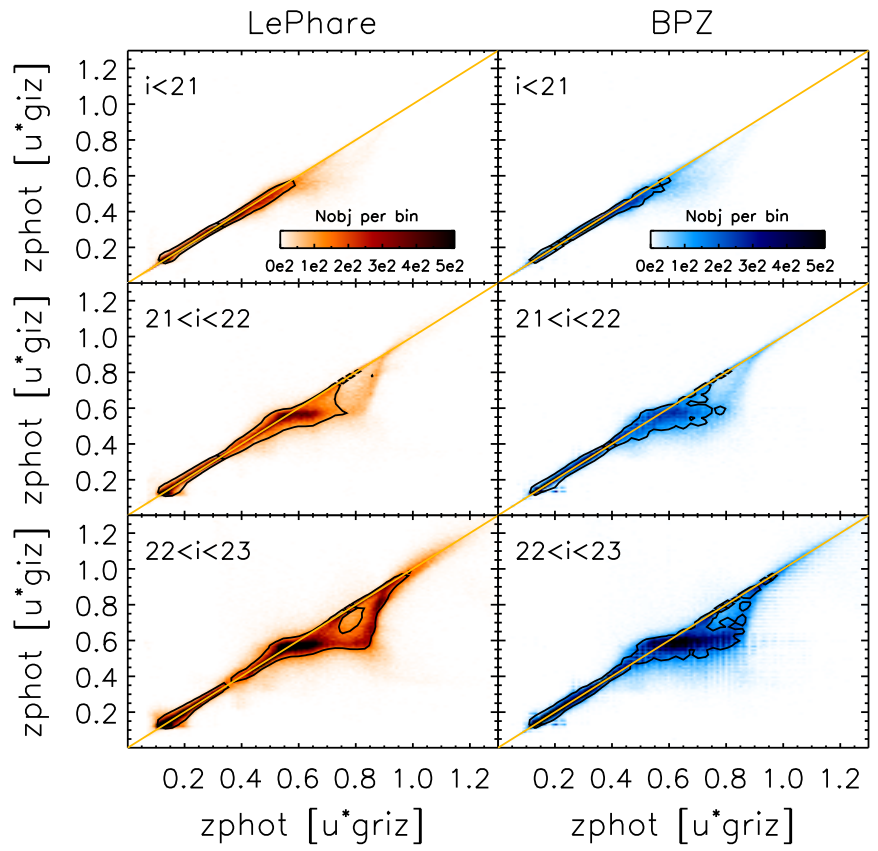

Figure 15. Photo-z's with $u^{*}$ giz bands vs. photo-z's with $u^{*}$ griz bands for our NGVSLenS/phot23 photometric sample by magnitude bins. Left: photo-z's estimated with Le Phare; right: photo- $z$ 's estimated with BPZ. We represent the density of objects per $0.01 \times 0.01$ bin in $z_{\text {phot }}$. Black contours represent the loci enclosing $68 \%$ of the distribution. The analysis with a large NGVSLenS photometric sample confirms the one done with the CFHTLenS spectroscopic sample.

(A color version of this figure is available in the online journal.)

\subsection{Joint Analysis of Photo-z Dependence on Magnitude and Redshift}

The above analysis shows that the photo-z's statistics can be biased by the properties of the spectroscopic sample chosen as reference. Indeed, the galaxies selected as targets for spectroscopic samples might have properties that are not characteristic of the entire photometric sample. In particular, spectroscopic samples are dominated by brighter galaxies that represent a small percentage of the entire photometric samples at a given 


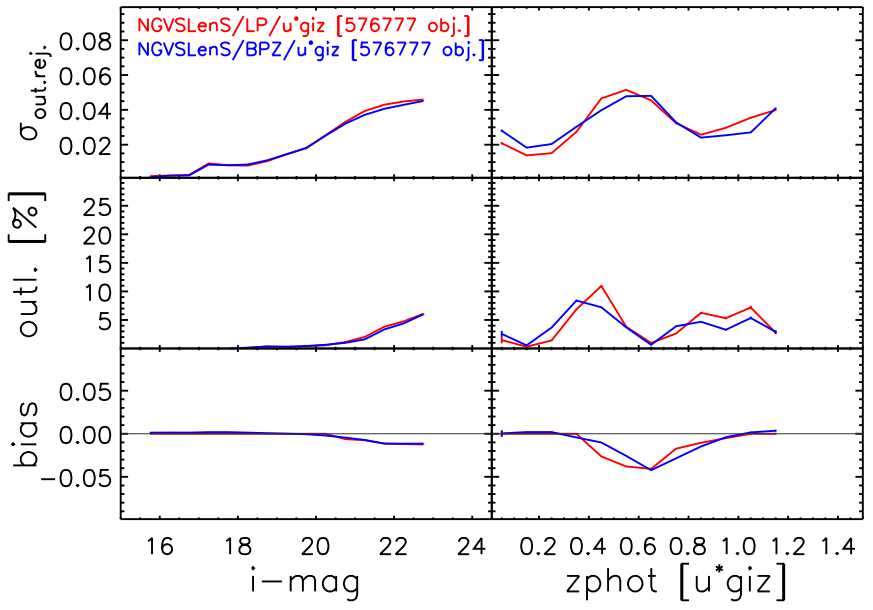

Figure 16. Statistics for photo-z's (estimated with $u^{*} g i z$ bands) in our NGVSLenS/phot23 photometric sample $\left(>5 \times 10^{5}\right.$ objects $)$ as a function of magnitude (left) and redshift (right). Photo-z's estimated with Le Phare are in red, and those estimated with BPZ are in blue. Figure is similar to Figure 13, but the spectroscopic sample has been replaced by a NGVSLenS photometric sample $\left(5.8 \times 10^{5}\right.$ objects $)$, and the spec- $z$ 's have been replaced by the photo- $z$ 's estimated with the $u^{*}$ griz bands. Error bars, calculated assuming a Poissonian distribution, are not visible because of the large size of the sample. The analysis with a large NGVSLenS photometric sample confirms the one done with the CFHTLenS spectroscopic sample.

(A color version of this figure is available in the online journal.)

redshift. This means that fainter galaxies, which statistically dominate the photometric sample, will not be correctly represented in the spectroscopic sample. In our case, we do have spectroscopic samples that cover the fainter magnitudes (see Figure 6, bottom); however, at a given redshift they include significantly fewer galaxies than those that cover the bright end, which dominate our statistics shown in previous sections.

Moreover, as shown in Figure 6, our spectroscopic samples cover different ranges in redshift and magnitude, and, when binning only in magnitude or in redshift, we are mixing galaxies that have very different properties.

For those reasons, we here separate different magnitude bins at a given redshift and perform a joint analysis in enough small bins of redshift and magnitude, in which we can select galaxies with similar properties.

In Figure 17 and Table 5, we present a joint analysis in redshift and magnitude on the entire NGVSLenS and CFHTLenS spectroscopic samples ( $\sim 83,000$ galaxies). We have already discussed that Le Phare and BPZ provide photo-z's with similar properties, and, for simplicity, we present only Le Phare photo-z's for this analysis. When using BPZ, our results do not change.

This analysis clarifies what has been observed in the previous sections and refines the conclusions. In a given redshift bin, the quality of the photo- $z$ 's depends on magnitude, with fainter galaxies having more uncertain photo-z's estimates. Bright galaxies with $i<21$ mag have accurate photo- $z$ 's, independent of redshift. Their bias and scatter are low ( $\mid$ bias $\mid<0.02$ and $\left.\sigma_{\text {outl.rej. }}<0.04\right)$, and they have a small number of outliers $(<6 \%)$. This is also true when the photo- $z$ 's are estimated without the $r$ band for $i<20 \mathrm{mag}$. For fainter galaxies $(i>21 \mathrm{mag})$, it results in a steeper decline in the accuracy of the photo- $z$ estimate as a function of magnitude in the $0.3 \lesssim z_{\text {phot }} \lesssim 0.8$. As previously explained, this depends on tighter priors on the brightest galaxies, and on the predominance of galaxies with more defined $4000 \AA$ breaks at the higher end of the luminosity function.
At fixed magnitude, photo- $z$ estimates at higher redshift are often more accurate, just because we are probing galaxies with higher absolute luminosity, e.g., intrinsically brighter galaxies at higher redshift, that will have more defined $4000 \AA$ breaks. When the optical bandpasses no longer bracket the $4000 \AA$ break $(z \gtrsim 1.2)$, this is no longer true and the photo- $z$ 's become more uncertain even for bright red sequence galaxies.

\section{ANGULAR CORRELATION FUNCTION}

A complementary way to test the photo- $z$ accuracy using the whole NGVSLenS sample is to calculate the galaxy angular correlation function, $(w(\theta)$; e.g., Newman 2008; Hildebrandt et al. 2009a; McQuinn \& White 2013), in different redshift bins. The advantage of this approach is that we probe the photo- $z$ directly on the NGVSLenS data and do not have to make assumptions about the spectroscopic samples we are using. This permits an estimation of the level of contamination between photometric redshift bins. As a result of galaxy clustering, the angular correlation in a given redshift bin (autocorrelation) should be positive on small scales when compared to a random distribution of points; on large scales, the angular autocorrelation should tend to zero. When looking at two redshift bins, the angular correlation (cross-correlation) should be zero if the redshift bins are well-separated because the galaxies are physically separated by large distances; if the considered redshift bins are close to each other and have sizes close to the typical photo- $z$ uncertainty, this produces a nonzero cross-correlation. We refer to Erben et al. (2009) for a detailed presentation of the angular correlation function formalism.

We apply pairwise analysis using the publicly available athena ${ }^{39}$ tree code on our NGVSLenS data in redshift bins defined by the following limits: $z_{\text {cut }}=$ $0.1 ; 0.2 ; 0.3 ; 0.4 ; 0.5 ; 0.6 ; 0.7 ; 0.8 ; 0.9 ; 1.0 ; 1.2$. Note that we neglect the effects of magnification (Scranton et al. 2005; Hildebrandt et al. 2009b). We do not consider the range $0 \leqslant z<0.1$, as it contains too few objects (a few thousands) to estimate robust statistics. We only consider objects having $i<23$ mag, not classified as a star or GC (see Appendix C). To exclude galaxies with unreliable $z_{\text {phot }}$, we exclude galaxies with $z_{\text {phot,err. }}>0.25$ (according to Figure 11, the $3 \sigma$ upper limit of $z_{\text {photerr. at }} i=$ $23 \mathrm{mag}$ is 0.25 ). Also excluding all masked areas and field edges (to prevent duplicates when merging the fields), we end up with $4 \times 10^{5}$ objects when using photo- $z$ 's derived from $u^{*}$ griz bands and $1.1 \times 10^{6}$ objects with just $u^{*}$ giz filters. To compare with a random distribution, we generate random catalogs, having uniformly distributed positions with the same geometry as our NGVSLenS data (imaged areas and masks). Because our chosen bin widths are at worst about twice as large as our photo- $z$ scatter $\left(\sigma_{\text {outl.rej. }} \lesssim 0.06\right)$, we expect a low-level cross-correlation signal in neighboring redshift bins and a $w(\theta)$ compatible with zero for bins distant in redshift.

Figure 18 shows $w(\theta)$ for the photo- $z$ 's estimated with the $u^{*}$ griz bands and Le Phare. The figure-hence the conclusions-is similar if we use BPZ or if we use the $u^{*} g i z$ bands. This figure is consistent with the expected behavior, which is a positive signal for autocorrelation (diagonal panels, in blue) and a signal consistent with zero for cross-correlation (red panels), except for adjacent redshift windows (second panel of each line, rightward of the blue panels) and for the second off-diagonal panels at small scales (third panel of each line).

\footnotetext{
39 http://cosmostat.org/athena.html
} 

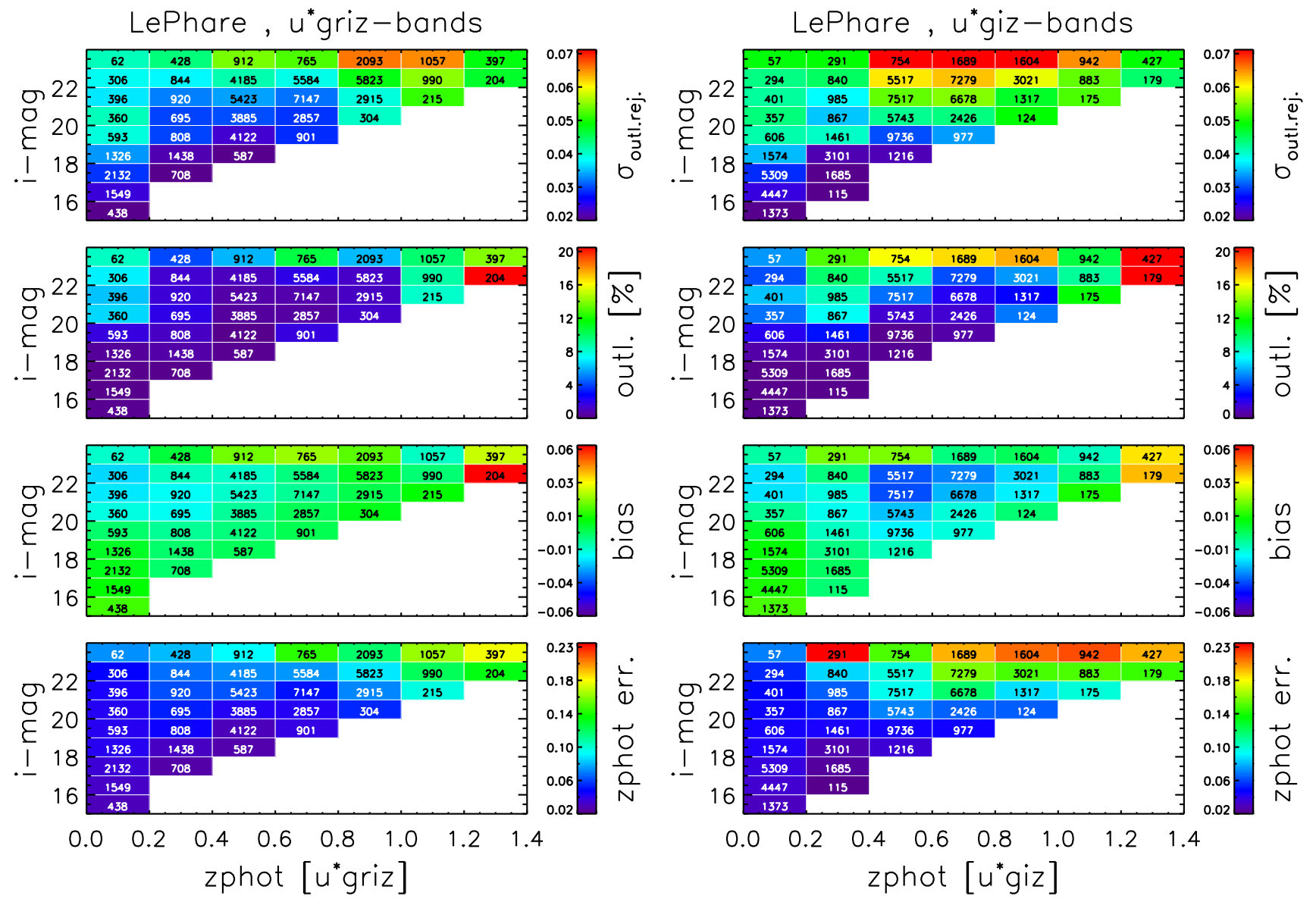

Figure 17. Joint analysis of our photo-z's estimated with Le Phare and the $u^{*}$ griz bands (left) and the $u^{*} g i z$ bands (right). From top to bottom, we display $\sigma_{\text {outl.rej. }}$, outl., bias, and the median value of $z_{\text {phot,err. }}$. We use here our full spectroscopic sample (NGVSLenS and CFHTLenS; $\sim 83,000$ galaxies). The statistics are presented by binning it both in magnitude and photo- $z$. We only display the bins where we have more than 50 galaxies, and for those bins, we report the number of galaxies used to compute the statistics.

(A color version of this figure is available in the online journal.)

A signal consistent with zero for cross-correlation in distant redshift bins confirms that our levels of contamination are minimal, as found in our previous analysis with spectroscopic samples.

\section{CONCLUSIONS}

We present an analysis of the determination of the photo- $z$ catalog for the NGVS survey. This survey images $104 \mathrm{deg}^{2}$ around the Virgo cluster with the $u^{*}$ giz bands, among which 34 pointings have $r$-band coverage. To obtain good quality matched photometry, we used an upgraded version of the THELI pipeline developed for the analysis of the CFHTLenS data, which have properties very similar to our data. The THELI pipeline products are the coadded astrometrically and photometrically calibrated images for all the NGVSLenS pointings in each filter, the photometric catalogs, and the photo- $z$ catalogs.

We uniformly calibrated the photometry using the SDSS, which covers the full NGVSLenS. We built photometric catalogs from the multiwavelength images convolved to the same seeing on each field. This PSF homogenization allows an accurate measurement of colors, which is fundamental to obtain precise photo- $z$ 's. We paid particular attention to the magnitude uncertainty estimation, accounting for the convolution process. We estimate the photo-z's with two template-fitting codes, $L e$ Phare and BPZ. We extended the prior of those codes to bright objects (seven magnitudes brighter) and are thus able to accurately estimate photo- $z$ 's over a large range of magnitudes.
To assess the quality of our photo- $z$ catalog, we used a large spectroscopic sample $(\sim 83,000$ galaxies $)$ in the $0.01 \leqslant z_{\text {spec }}<$ 1.5 and $15.5 \mathrm{mag} \leqslant i \leqslant 24.5 \mathrm{mag}$ ranges. We presented a detailed analysis of our photo-z's as a function of the measured magnitude, redshift, and the number of bandpasses used for their estimation $\left(u^{*}\right.$ griz or $\left.u^{*} g i z\right)$.

Our analysis concluded that both codes perform very similarly. When using the $u^{*}$ griz bands, we obtain accurate photo-z's for $i \lesssim 23$ mag or $z_{\text {phot }} \lesssim 1$ : the bias is reasonable $(|b i a s|<0.02)$, the scatter $\sigma_{\text {outl.rej }}$ increases with the magnitude (from 0.02 to 0.05 for $i$ in $[15.5,23]$ ), and the outliers represent less than 5\% of the sample. For photo-z's estimated without the $r$ band (i.e., with the $u^{*} g i z$ bands), the accuracy decreases slightly. The lack of $r$-band results in more pronounced uncertainties in the $0.3 \lesssim z_{\text {phot }} \lesssim 0.8$ range, where it samples the $4000 \AA$ break. In this redshift range, we have $-0.05<$ bias $<-0.02$, $\sigma_{\text {out.rej }} \sim 0.06$ and an outlier rate that peaks at $10 \%-15 \%$. The quality of photo-z's estimated with the $u^{*}$ giz bands also decreases at $i>21 \mathrm{mag}$. However, we remark that the brightest galaxies, e.g., the LRGs, which constitute the main part of our NGVSLenS spectroscopic sample at $0.3<z_{\text {spec }}<0.8$, have photo- $z$ 's of almost similar quality to when the $r$ band is used (but a slightly higher bias). This is because of the different typical magnitudes of the LRGs with respect to the average galaxy in a given redshift bin: these galaxies have tighter priors because they are the brightest at a given redshift and have a 
Table 5

Photo-z (Estimated with Le Phare) Properties for Our Compiled Spectroscopic Sample (NGVSLenS and CFHTLenS)

\begin{tabular}{|c|c|c|c|c|c|c|c|c|c|c|c|c|c|c|c|}
\hline \multicolumn{4}{|c|}{ Binning } & \multicolumn{6}{|c|}{$u^{*}$ griz Bands } & \multicolumn{6}{|c|}{$u^{*} g i z$ Bands } \\
\hline $\begin{array}{l}i^{\min } \\
(\mathrm{mag})\end{array}$ & $\begin{array}{c}i^{\max } \\
(\mathrm{mag})\end{array}$ & $z_{\mathrm{phot}}^{\min }$ & $z_{\text {phot }}^{\max }$ & bias & $\sigma_{\text {outl.rej. }}$ & $\begin{array}{c}\text { outl. } \\
(\%)\end{array}$ & $z_{\text {phot,err. }}$ & Ngal & zSurvey $^{\mathrm{a}}$ & bias & $\sigma_{\text {outl.rej. }}$ & $\begin{array}{c}\text { outl. } \\
(\%)\end{array}$ & $z_{\text {phot,err. }}$ & Ngal & zSurvey $^{\mathrm{a}}$ \\
\hline 15.0 & 16.0 & 0.0 & 0.2 & 0.01 & 0.02 & 0 & 0.03 & 438 & A & 0.02 & 0.02 & 0 & 0.03 & 1373 & A \\
\hline 16.0 & 17.0 & 0.0 & 0.2 & 0.01 & 0.02 & 0 & 0.03 & 1549 & A & 0.01 & 0.02 & 0 & 0.03 & 4447 & A \\
\hline 16.0 & 17.0 & 0.2 & 0.4 & $\ldots$ & $\ldots$ & $\ldots$ & $\ldots$ & $\ldots$ & $\ldots$ & 0.00 & 0.02 & 0 & 0.02 & 115 & A \\
\hline 17.0 & 18.0 & 0.0 & 0.2 & 0.01 & 0.03 & 0 & 0.04 & 2132 & A & 0.01 & 0.03 & 0 & 0.04 & 5309 & A \\
\hline 17.0 & 18.0 & 0.2 & 0.4 & 0.00 & 0.02 & 0 & 0.03 & 708 & $\mathrm{D}$ & 0.00 & 0.02 & 0 & 0.02 & 1685 & $\mathrm{D}$ \\
\hline 18.0 & 19.0 & 0.0 & 0.2 & 0.00 & 0.03 & 0 & 0.04 & 1326 & $\mathrm{~B}$ & 0.01 & 0.03 & 1 & 0.04 & 1574 & B \\
\hline 18.0 & 19.0 & 0.2 & 0.4 & 0.00 & 0.02 & 1 & 0.03 & 1438 & $\mathrm{D}$ & -0.01 & 0.02 & 0 & 0.03 & 3101 & $\mathrm{D}$ \\
\hline 18.0 & 19.0 & 0.4 & 0.6 & 0.00 & 0.02 & 0 & 0.03 & 587 & $\mathrm{D}$ & -0.01 & 0.02 & 0 & 0.04 & 1216 & $\mathrm{D}$ \\
\hline 19.0 & 20.0 & 0.0 & 0.2 & 0.00 & 0.04 & 2 & 0.04 & 593 & $\mathrm{C}$ & 0.00 & 0.04 & 2 & 0.04 & 606 & $\mathrm{C}$ \\
\hline 19.0 & 20.0 & 0.2 & 0.4 & -0.01 & 0.03 & 1 & 0.05 & 808 & $\mathrm{D}$ & -0.01 & 0.04 & 3 & 0.04 & 1461 & $\mathrm{D}$ \\
\hline 19.0 & 20.0 & 0.4 & 0.6 & 0.00 & 0.02 & 0 & 0.03 & 4122 & $\mathrm{D}$ & -0.02 & 0.03 & 0 & 0.05 & 9736 & $\mathrm{D}$ \\
\hline 19.0 & 20.0 & 0.6 & 0.8 & 0.00 & 0.03 & 2 & 0.04 & 901 & D & -0.01 & 0.03 & 1 & 0.05 & 977 & D \\
\hline 20.0 & 21.0 & 0.0 & 0.2 & -0.01 & 0.04 & 6 & 0.04 & 360 & $\mathrm{E}$ & -0.01 & 0.04 & 5 & 0.05 & 357 & $\mathrm{E}$ \\
\hline 20.0 & 21.0 & 0.2 & 0.4 & -0.02 & 0.03 & 2 & 0.05 & 695 & $\mathrm{H}$ & -0.02 & 0.04 & 7 & 0.05 & 867 & $\mathrm{H}$ \\
\hline 20.0 & 21.0 & 0.4 & 0.6 & -0.01 & 0.03 & 0 & 0.04 & 3885 & $\mathrm{~F}$ & -0.02 & 0.04 & 1 & 0.07 & 5743 & $\mathrm{~F}$ \\
\hline 20.0 & 21.0 & 0.6 & 0.8 & 0.00 & 0.03 & 1 & 0.04 & 2857 & $\mathrm{~F}$ & -0.01 & 0.04 & 1 & 0.07 & 2426 & $\mathrm{~F}$ \\
\hline 20.0 & 21.0 & 0.8 & 1.0 & 0.01 & 0.04 & 1 & 0.06 & 304 & $\mathrm{~F}$ & 0.00 & 0.05 & 5 & 0.07 & 124 & $\mathrm{~F}$ \\
\hline 21.0 & 22.0 & 0.0 & 0.2 & -0.02 & 0.04 & 6 & 0.04 & 396 & $\mathrm{H}$ & -0.02 & 0.04 & 6 & 0.05 & 401 & $\mathrm{E}$ \\
\hline 21.0 & 22.0 & 0.2 & 0.4 & -0.02 & 0.03 & 2 & 0.06 & 920 & $\mathrm{E}$ & -0.01 & 0.04 & 8 & 0.07 & 985 & $\mathrm{H}$ \\
\hline 21.0 & 22.0 & 0.4 & 0.6 & -0.01 & 0.03 & 1 & 0.06 & 5423 & $\mathrm{~F}$ & -0.04 & 0.05 & 5 & 0.10 & 7517 & $\mathrm{~F}$ \\
\hline 21.0 & 22.0 & 0.6 & 0.8 & -0.01 & 0.03 & 1 & 0.05 & 7147 & $\mathrm{~F}$ & -0.02 & 0.05 & 2 & 0.12 & 6678 & $\mathrm{~F}$ \\
\hline 21.0 & 22.0 & 0.8 & 1.0 & 0.00 & 0.04 & 1 & 0.07 & 2915 & $\mathrm{~F}$ & -0.02 & 0.05 & 3 & 0.09 & 1317 & $\mathrm{~F}$ \\
\hline 21.0 & 22.0 & 1.0 & 1.2 & 0.01 & 0.05 & 8 & 0.10 & 215 & $\mathrm{~F}$ & 0.01 & 0.05 & 11 & 0.10 & 175 & $\mathrm{~F}$ \\
\hline 22.0 & 23.0 & 0.0 & 0.2 & -0.02 & 0.04 & 7 & 0.05 & 306 & $\mathrm{H}$ & -0.02 & 0.04 & 4 & 0.05 & 294 & $\mathrm{H}$ \\
\hline 22.0 & 23.0 & 0.2 & 0.4 & -0.01 & 0.04 & 1 & 0.07 & 844 & $\mathrm{H}$ & 0.00 & 0.04 & 10 & 0.09 & 840 & $\mathrm{H}$ \\
\hline 22.0 & 23.0 & 0.4 & 0.6 & -0.01 & 0.04 & 1 & 0.07 & 4185 & $\mathrm{~F}$ & -0.03 & 0.06 & 8 & 0.10 & 5517 & $\mathrm{~F}$ \\
\hline 22.0 & 23.0 & 0.6 & 0.8 & 0.00 & 0.04 & 2 & 0.08 & 5584 & $\mathrm{~F}$ & -0.03 & 0.06 & 4 & 0.16 & 7279 & $\mathrm{~F}$ \\
\hline 22.0 & 23.0 & 0.8 & 1.0 & 0.01 & 0.05 & 1 & 0.09 & 5823 & $\mathrm{~F}$ & -0.02 & 0.06 & 5 & 0.15 & 3021 & $\mathrm{~F}$ \\
\hline 22.0 & 23.0 & 1.0 & 1.2 & 0.00 & 0.05 & 9 & 0.12 & 990 & $\mathrm{~F}$ & 0.00 & 0.05 & 9 & 0.15 & 883 & $\mathrm{~F}$ \\
\hline 22.0 & 23.0 & 1.2 & 1.4 & 0.06 & 0.05 & 32 & 0.14 & 204 & $\mathrm{~F}$ & 0.04 & 0.04 & 31 & 0.15 & 179 & $\mathrm{~F}$ \\
\hline 23.0 & 24.0 & 0.0 & 0.2 & -0.01 & 0.04 & 8 & 0.07 & 62 & $\mathrm{H}$ & -0.01 & 0.05 & 5 & 0.07 & 57 & $\mathrm{H}$ \\
\hline 23.0 & 24.0 & 0.2 & 0.4 & 0.00 & 0.04 & 4 & 0.08 & 428 & $\mathrm{G}$ & 0.02 & 0.05 & 13 & 0.64 & 291 & $\mathrm{H}$ \\
\hline 23.0 & 24.0 & 0.4 & 0.6 & 0.02 & 0.05 & 6 & 0.09 & 912 & $\mathrm{H}$ & 0.02 & 0.07 & 16 & 0.15 & 754 & $\mathrm{H}$ \\
\hline 23.0 & 24.0 & 0.6 & 0.8 & 0.02 & 0.05 & 10 & 0.15 & 765 & $\mathrm{H}$ & 0.00 & 0.07 & 16 & 0.20 & 1689 & $\mathrm{H}$ \\
\hline 23.0 & 24.0 & 0.8 & 1.0 & 0.02 & 0.06 & 6 & 0.12 & 2093 & $\mathrm{H}$ & 0.00 & 0.07 & 17 & 0.21 & 1604 & $\mathrm{H}$ \\
\hline 23.0 & 24.0 & 1.0 & 1.2 & -0.01 & 0.06 & 10 & 0.17 & 1057 & $\mathrm{H}$ & -0.01 & 0.06 & 10 & 0.22 & 942 & $\mathrm{H}$ \\
\hline 23.0 & 24.0 & 1.2 & 1.4 & 0.03 & 0.05 & 14 & 0.18 & 397 & $\mathrm{H}$ & 0.04 & 0.05 & 20 & 0.20 & 427 & $\mathrm{H}$ \\
\hline
\end{tabular}

Notes. We report quantities only for the bins where we have more than 50 galaxies.

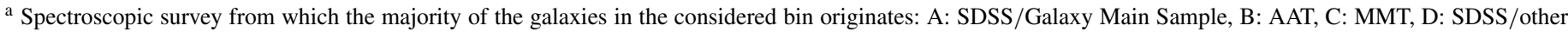
programs, E: VVDS/F22, F: VIPERS, G: VVDS/F02, and H: DEEP2/EGS.

well-defined $4000 \AA$ A break. Our results are visualized and interpreted in a joint analysis in magnitude and redshift bins.

Finally, we presented an analysis of the angular correlation function $w(\theta)$ to internally assess the quality of our photo- $z$ 's using the whole NGVSLenS sample with $i \leqslant 24 \mathrm{mag}$ and $0.1 \leqslant z_{\text {phot }} \leqslant 1.2$. We obtain results that are consistent with expectations, i.e., a positive signal for autocorrelation (decreasing with increasing angle) and a signal consistent with zero for cross-correlation when considering redshift bins with a redshift separation greater than 0.1 .

The NGVSLenS catalogs will be made public on 2015 June 1 on the NGVS Web site. ${ }^{40}$ Before that date, please contact us if you would like to use them. ${ }^{41}$

\footnotetext{
40 https://www.astrosci.ca/NGVS/The_Next_Generation_Virgo_Cluster_ Survey/Home.html

41 simona.mei@obspm.fr
}

We thank the anonymous referee for careful reading and suggestions, which improved the clarity of the paper. The French authors acknowledge the support of the French Agence Nationale de la Recherche (ANR) under the reference ANR10BLANC-0506-01-Projet VIRAGE. S.M. acknowledges financial support from the Institut Universitaire de France (IUF). H.H. is supported by the DFG Emmy Noether grant Hi 1495/2-1. C.L. acknowledges support from the National Natural Science Foundation of China (grants 11203017, 11125313, and 10973028). R.P.M. acknowledges support from FONDECYT Postdoctoral Fellowship Project 3130750. E.W.P. acknowledges support from the National Natural Science Foundation of China under grant 11173003 and from the Strategic Priority Research Program, "The Emergence of Cosmological Structures," of the Chinese Academy of Sciences, grant XDB09000105. T.H.P. acknowledges support from FONDECYT Regular Grant (1121005) and BASAL Center for Astrophysics and Associated Technologies (PFB-06). H.Z. acknowledges support from 


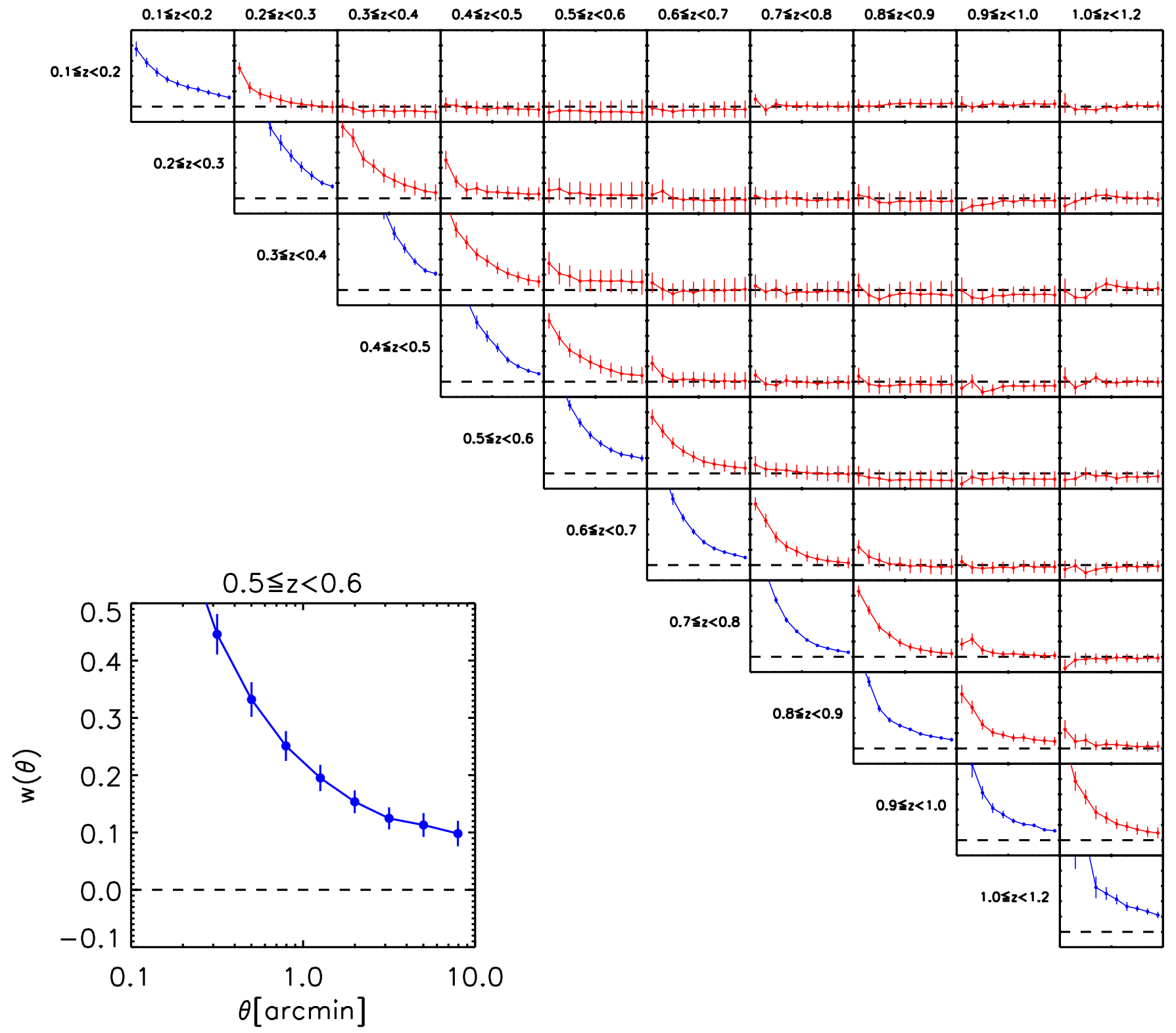

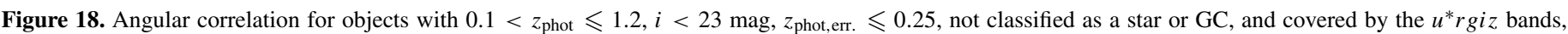

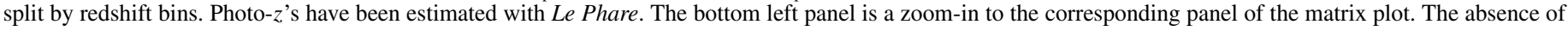
positive angular correlation in distant redshift bins is consistent with independent photometric redshift measurements.

(A color version of this figure is available in the online journal.)

the China-CONICYT Postdoctoral Fellowship, administered by the Chinese Academy of Sciences South America Center for Astronomy (CASSACA). A.R. thanks Begoña Ascaso for useful discussions.

This work is based on observations obtained with MegaPrime/MegaCam, a joint project of CFHT and CEA/ DAPNIA, at the Canada-France-Hawaii Telescope (CFHT), which is operated by the National Research Council (NRC) of Canada, the Institut National des Sciences de l'Univers of the Centre National de la Recherche Scientifique (CNRS) of France, and the University of Hawaii. This research used the facilities of the Canadian Astronomy Data Centre operated by the National Research Council of Canada with the support of the Canadian Space Agency. This publication has made use of data products from SDSS-III (full text acknowledgment is at http://www.sdss3.org/collaboration/boiler-plate.php). Funding for the DEEP2 survey has been provided by NSF grants AST9509298, AST-0071048, AST-0071198, AST-0507428, and AST0507483, as well as NASA LTSA grant NNG04GC89G. Some of the data presented herein were obtained at the W. M. Keck Observatory, which is operated as a scientific partnership among the California Institute of Technology, the University of California, and the National Aeronautics and Space Administration. The Observatory was made possible by the generous financial support of the W. M. Keck Foundation. The authors wish to recognize and acknowledge the very significant cultural role and reverence that the summit of Mauna Kea has always had within the indigenous Hawaiian community. We are most fortunate to have the opportunity to conduct observations from this mountain. This research uses data from the VIMOS VLT Deep Survey, obtained from the VVDS database operated by Cesam, Laboratoire d'Astrophysique de Marseille, France. This paper 
Table 6

Prior Used in This Article

\begin{tabular}{|c|c|c|c|c|c|c|}
\hline $\begin{array}{l}\text { Spectral } \\
\text { Template Type } T\end{array}$ & $\begin{array}{c}i_{\text {ref }} \\
(\mathrm{mag})\end{array}$ & $\alpha_{t}$ & $z_{0 t}$ & $k_{m t}$ & $f_{t}$ & $k_{t}$ \\
\hline \multicolumn{7}{|c|}{$i_{\mathrm{AB}} \leqslant 12.5 \mathrm{mag}$} \\
\hline \multicolumn{7}{|c|}{$p(z) \propto 1$ if $z<0.1, p(z)=0$ else } \\
\hline \multicolumn{7}{|c|}{$12.5 \mathrm{mag}<i_{\mathrm{AB}} \leqslant 17 \mathrm{mag}$} \\
\hline Ell & 12.5 & 2.46 & 0 & 0.027 & 0.86 & 0.062 \\
\hline Spi & 12.5 & 2.07 & 0 & 0.021 & 0.14 & -0.108 \\
\hline Irr & 12.5 & 1.89 & 0 & 0.015 & $\ldots$ & $\ldots$ \\
\hline \multicolumn{7}{|c|}{$17 \mathrm{mag}<i_{\mathrm{AB}} \leqslant 20 \mathrm{mag}$} \\
\hline Ell & 17.0 & 2.46 & 0.121 & 0.103 & 0.65 & 0.257 \\
\hline Spi & 17.0 & 1.94 & 0.095 & 0.098 & 0.23 & -0.014 \\
\hline Irr & 17.0 & 1.95 & 0.069 & 0.077 & $\ldots$ & $\ldots$ \\
\hline \multicolumn{7}{|c|}{$20 \mathrm{mag}<i_{\mathrm{AB}}$} \\
\hline Ell & 20.0 & 2.46 & 0.431 & 0.091 & 0.30 & 0.40 \\
\hline Spi & 20.0 & 1.81 & 0.390 & 0.100 & 0.35 & 0.30 \\
\hline Irr & 20.0 & 2.00 & 0.300 & 0.150 & $\ldots$ & $\ldots$ \\
\hline
\end{tabular}

uses data from the VIMOS Public Extragalactic Redshift Survey (VIPERS). VIPERS has been performed using the ESO Very Large Telescope, under the "Large Programme" 182.A0886. The participating institutions and funding agencies are listed at http://vipers.inaf.it.

\section{APPENDIX A}

\section{PRIOR EXTENSION TO BRIGHT OBJECTS}

Le Phare and BPZ were designed for high-redshift studies. Both codes use similar priors for $i>20$ mag galaxies (calibrated with $\sim 1300$ galaxies for BPZ and with $\sim 6500$ galaxies for $L e$ Phare), and for $i<20$ mag a prior that is not calibrated on observed data. As a result of the large area covered by the NGVSLenS, $i<20$ mag galaxies represent a nonnegligible fraction of our sample. We thus build a new prior calibrated for bright and faint objects. Our estimated parameters are displayed in Table 6.

According to the formalism introduced in Benítez (2000) and using our template set (see Section 4.2 and Table 3), for a galaxy with an $i$-band magnitude $i_{\mathrm{AB}}$, the a priori probability of having a redshift $z$ and a spectral template type $T$ is

$$
p\left(z, T \mid i_{\mathrm{AB}}\right)=p\left(T \mid i_{\mathrm{AB}}\right) \times p\left(z \mid T, i_{\mathrm{AB}}\right) .
$$

$p\left(T \mid i_{\mathrm{AB}}\right)$ is the probability for a galaxy of magnitude $i_{\mathrm{AB}}$ to have a spectral template type $T$ and is parameterized as

$$
p\left(T \mid i_{\mathrm{AB}}\right) \propto f_{t} \times \exp \left(-k_{t} \times\left[i_{\mathrm{AB}}-i_{\mathrm{ref}}\right]\right) .
$$

$p\left(z \mid T, i_{\mathrm{AB}}\right)$ is the probability for a galaxy of magnitude $i_{\mathrm{AB}}$ and spectral template type $T$ to have a redshift $z$ and is parameterized as

$$
p\left(z \mid T, i_{\mathrm{AB}}\right) \propto z^{\alpha_{t}} \times \exp \left(-\left[\frac{z}{z_{0 t}+k_{m t} \times\left(i_{\mathrm{AB}}-i_{\mathrm{ref}}\right)}\right]^{\alpha_{t}}\right) .
$$

$i_{\text {ref }}$ is a reference magnitude.

For galaxies with $i>20 \mathrm{mag}$, we use the Le Phare prior, calibrated on the robust VVDS spectroscopic sample. For galaxies with $12.5 \mathrm{mag}<i \leqslant 17 \mathrm{mag}$, we calibrate

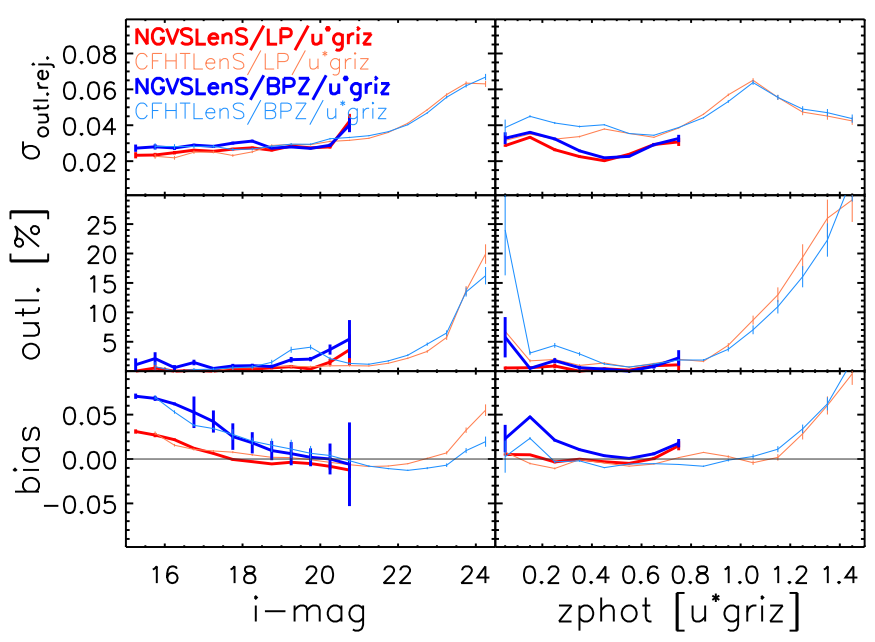

Figure 19. Statistics for photo- $z$ 's (estimated with $u^{*}$ griz bands) as a function of magnitude (left) and redshift (right), when using Le Phare and BPZ original priors. Symbols are similar to those in Figure 9. At $i<20 \mathrm{mag}$, the bias is larger than when using our recomputed prior in Figure 9.

(A color version of this figure is available in the online journal.)

our prior with the SDSS spectroscopic Galaxy Main Sample (York et al. 2000; Strauss et al. 2002), using the DR10 release (Ahn et al. 2014). We select objects with class=GALAXY and use cModelMag_i as $i$-band total magnitude and ModelMag quantities to compute colors (we correct for extinction). This sample is complete down to $r \leqslant 17.77 \mathrm{mag}$ and thus complete down to $i \sim 17 \mathrm{mag}$ (in fact, most of the galaxies with $r \leqslant 17.77$ mag have $z \lesssim 0.4$, and for $z \lesssim 0.4$, the color $(r-i) \lesssim$ 0.8 ; see Figure 5). For consistency with standard prior already implemented in BPZ and Le Phare, we follow the formalism of Benítez (2000) and define three broad spectral classes: Ellipticals (Ell template), Spirals (Sbc and Scd templates), and Irregulars (Im, SB2, and SB3 templates). We associate each galaxy from the SDSS spectroscopic Galaxy Main Sample to one of those broad spectral classes by using the best-fit template (from the photometric redshift code) when fixing the redshift at $z_{\text {spec }}$. We thus have $\sim 320,000$ galaxies with $12.5 \mathrm{mag}<$ $i \leqslant 17$ mag and classified into three broad spectral classes. We then fit with a least-square fitting method (IDL MPFIT package, Markwardt 2009) the fraction of each spectral type as a function of the magnitude with Equation (A2) and obtain the $k_{t}$ parameter and the redshift distribution of each spectral type $\mathrm{T}$ in each magnitude bin with Equation (A3) and obtain the $\alpha_{t}, z_{0 t}$, and $k_{m t}$ parameters. For $17 \mathrm{mag}<i \leqslant 20$ mag galaxies, we extrapolate the parameter values to match those fitted at $i_{\mathrm{AB}}=17 \mathrm{mag}$ and $i_{\mathrm{AB}}=20 \mathrm{mag}$. For $\alpha_{t}$, we take the mean value of those estimated at $i_{\mathrm{AB}}=17 \mathrm{mag}$ and $i_{\mathrm{AB}}=20 \mathrm{mag}$ and set the other parameters so that the quantities $z_{0 t}+k_{m t} \times\left(i_{\mathrm{AB}}-i_{\text {ref }}\right)$ and $f_{t} \times \exp \left(i_{\mathrm{AB}}-i_{\text {ref }}\right)$ are continuous. For $i \leqslant 12.5$ mag galaxies, we set a square prior, $p(z)$ being nonnull for $z<0.1$ and null for $z \geqslant 0.1$.

To illustrate how our new prior improves the photo- $z$ accuracy, we display in Figure 19 the statistics for the photo- $z$ estimated with $u^{*}$ griz bands and using Le Phare and BPZ original priors. When comparing with the Figure 9, we see that our new prior, for $i<20$ mag galaxies, improves significantly the bias and, to a lesser extent, the scatter and the percentage of outliers; as a consequence, it also improves the photo- $z$ accuracy at $z_{\text {phot }}<0.4$. 


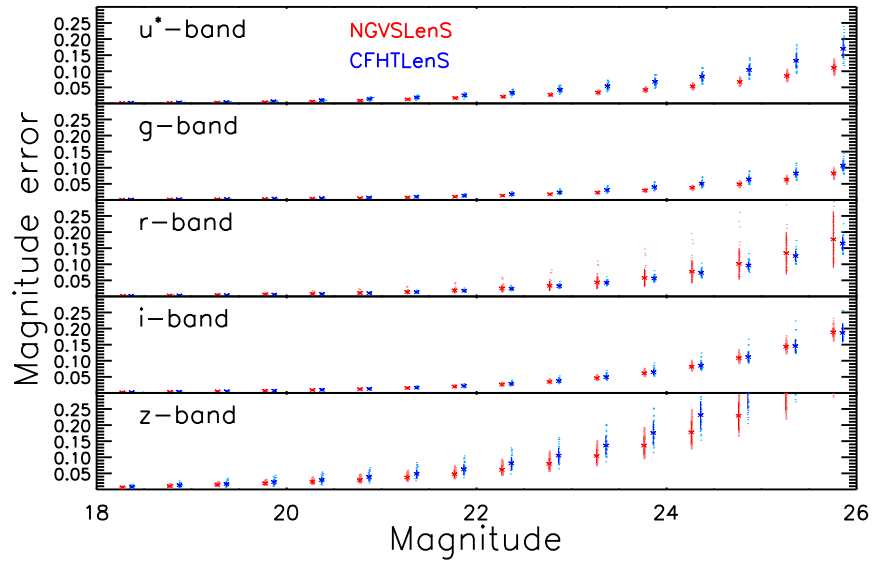

Figure 20. Magnitude uncertainty as a function of magnitude for the NGVSLenS (red) and the CFHTLenS (blue) data and for the $u^{*}$ griz bands. Each point represent the median values for a given field (i.e., $1 \mathrm{deg}^{2}$ ). The crosses and error bars represent the global values for each survey (median and standard deviation of those individual-i.e., for each field-data). CFHTLenS data are offset by 0.1 mag along the $x$ axis to increase readability. Both data sets have very similar depths for all bands.

(A color version of this figure is available in the online journal.)

\section{APPENDIX B}

\section{SIMILARITY OF THE NGVSLenS AND CFHTLenS DATA SETS}

In Section 5, we assume that the CFHTLenS data setrereduced with our changes in the THELI pipeline-has similar properties to our NGVSLenS data sets. This is justified by the fact that both data sets have been obtained with the same telescope, instrument, and filterset, have by construction similar depth, and have been reduced with the same pipeline.

In this section, we illustrate a posteriori the similarity of the data sets resulting from these two surveys. We display in Figure 20 our estimated uncertainties in magnitude as a function of magnitude for both data sets. Each point represents the median value of the magnitude error for a given magnitude bin in each field. The crosses and error bars represent the global values for each survey (median and standard deviation of those individual-i.e., for each field—-data).

We observe that two data sets have indeed very similar depths for all bands. The NGVSLenS has slightly better photometry in the $u^{*}$ and $z$ bands. We note the large standard deviations for the $r$ band for the NGVSLenS that are due to the continuation of the NGVSLenS program for some fields with smaller exposure times (see Section 4.4 of Ferrarese et al. 2012).

\section{APPENDIX C}

\section{STAR AND GLOBULAR CLUSTER REMOVAL}

In the figures using photometric samples, we exclude objects that are most probably stars or GCs. We describe in this appendix the criteria we use to exclude those objects. For more detailed NGVS studies of stars and GCs, please refer to D. Lokhorst et al. (in preparation) and Durrell et al. (2014), respectively.

\section{C.1. Star Removal}

To separate stars from galaxies we follow a simple approach. We classify an object as a star if it satisfies: CLASS_STAR $>0.95$ and $i<21.5 \mathrm{mag}$, where CLASS_STAR is a SEXTRACTOR output quantifying the star/galaxy classification. To assess

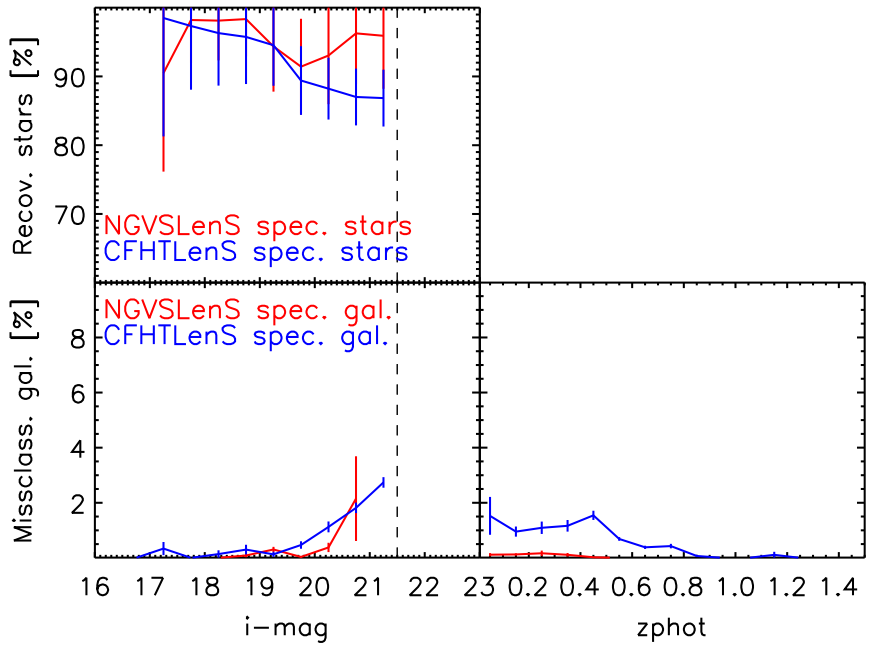

Figure 21. Star/galaxy separation efficiency as a function of the $i$ band magnitude (left) or $z_{\text {phot }}$ (right). The top panel represents the percentage of stars correctly classified as stars; the bottom panels represent the percentage of galaxies wrongly classified as stars. Please see the text for description of the samples. The vertical dashed lines show the faint limit $(i=21.5 \mathrm{mag})$ we use to assign the star classification. We report quantities only for the bins where we have more than 50 objects. Error bars are calculated assuming a Poissonian distribution.

(A color version of this figure is available in the online journal.)

the efficiency of our classification, we have in hand $\sim 6900$ CFHTLenS spectroscopically identified stars $(i=20.7 \pm$ $1.4 \mathrm{mag}$; using the DEEP2/EGS, VVDS, and VIPERS) and $\sim 3000$ NGVSLenS spectroscopic stars $(i=18.9 \pm 1.5 \mathrm{mag}$; using the SDSS), as well as our galaxy spectroscopic samples (NGVSLenS and CFHTLenS; $\sim 83,000$ galaxies with $0.01 \leqslant$ $\left.z_{\text {spec }}<1.5\right)$.

We present in Figure 21 the percentage of stars correctly classified as stars (top) and the percentage of galaxies misclassified as stars (bottom) when using our criteria. We correctly classify $>85 \%$ of the stars with $i<21.5 \mathrm{mag}$ and misclassify as stars $<5 \%$ of our galaxies. Note that, at $i=22 \mathrm{mag}$, we expect galaxies to be about 10 times more numerous than stars (see Figure 3 of Lilly et al. 2007, for the COSMOS field, which lies at a Galactic latitude lower than the NGVSLenS) and even more at fainter magnitudes. Therefore, for $i>21.5$ mag objects, the number of stars should be marginal when compared to the number of galaxies and should not affect the statistics. The performance of our star/galaxy classification is thus satisfactory for our needs.

\section{C.2. Globular Cluster Removal}

A special feature - and the main goal - of the NGVS is its full coverage of the Virgo cluster: a direct consequence is a massive detection of GCs. Whereas this provides a unique sample for Virgo science, these GCs may contaminate background science samples: in fact, as they are relatively small, red, and faint objects, they can easily be mistaken for higher-redshift objects.

We provide here the criteria we used to exclude GCs from our (photometric) samples in this study. The requirements, depending on the galaxy populations under study, are a tradeoff between maximizing the number of GCs removed and minimizing the number of background sources removed. For this paper, as we are not studying a special galaxy population, we aim at removing a significant portion of the GCs while accepting the loss of a marginal number of background sources. 
To test our method, we use our spectroscopic samples (NGVSLenS and CFHTLenS) with $0.01 \leqslant z_{\text {spec }}<1.5$ ( $\sim 83,000$ galaxies) for background sources and a sample of $\sim 750$ confirmed GCs around M87 (Hanes et al. 2001; Strader et al. 2011). As detailed in Section 5.1, our spectroscopic sample, being composite, fairly covers the different galaxy populations up to $z_{\text {spec }}<1.5$. After several tests, the following criteria gave the best results (according to our requirements), for removing GCs: (1) CLASS_STAR $>0.05$, (2) $18.0<$ MU_MAX $^{42}<21.5$, (3) $1.5<u-z<3.0$, (4) $z_{\text {phot }}<0.2$.

Applying these criteria results in the removal of $94 \%$ of the GCs and $0.2 \%$ of our spectroscopic sample (the vast majority of misclassified galaxies have $z_{\text {spec }}<0.25$ ). We note that these results do not depend on whether the photo-z's are estimated with Le Phare or BPZ or with the use of $u^{*}$ griz or $u^{*}$ giz bands. Our criteria to remove GCs also remove some stars (e.g., it removes $6 \%$ of our CFHTLenS spectroscopic stars) and vice versa. Although we are not able to properly separate stars and GCs, we are able to efficiently remove the vast majority of stars and GCs with a marginal loss of galaxies.

\section{REFERENCES}

Ahn, C. P., Alexandroff, R., Allende Prieto, C., et al. 2014, ApJS, 211, 17 Arnouts, S., Cristiani, S., Moscardini, L., et al. 1999, MNRAS, 310, 540 Arnouts, S., Moscardini, L., Vanzella, E., et al. 2002, MNRAS, 329, 355 Ball, N. M., Brunner, R. J., Myers, A. D., et al. 2008, ApJ, 683, 12 Baum, W. A. 1962, in IAU Symp. 15, Problems of Extra-Galactic Research, ed. G. C. McVittie (Cambridge: Cambridge Univ. Press), 390 Benítez, N. 2000, ApJ, 536, 571

Benítez, N., Ford, H., Bouwens, R., et al. 2004, ApJS, 150, 1

Bertin, E. 2006, in ASP Conf. Ser. 351, Astronomical Data Analysis Software and Systems XV, ed. C. Gabriel, C. Arviset, D. Ponz, \& S. Enrique (San Francisco, CA: ASP), 112

Bertin, E., \& Arnouts, S. 1996, A\&AS, 117, 393

Bertin, E., Mellier, Y., Radovich, M., et al. 2002, in ASP Conf. Ser. 281, Astronomical Data Analysis Software and Systems XI, ed. D. A. Bohlender, D. Durand, \& T. H. Handley (San Francisco, CA: ASP), 228

Bielby, R., Hudelot, P., McCracken, H. J., et al. 2012, A\&A, 545, A23

Bolzonella, M., Miralles, J., \& Pelló, R. 2000, A\&A, 363, 476

Boselli, A., Boissier, S., Heinis, S., et al. 2011, A\&A, 528, A107

Boulade, O., Charlot, X., Abbon, P., et al. 2003, Proc. SPIE, 4841, 72

Brammer, G. B., van Dokkum, P. G., \& Coppi, P. 2008, ApJ, 686, 1503

Brodwin, M., Brown, M. J. I., Ashby, M. L. N., et al. 2006, ApJ, 651,791

Capak, P., Cowie, L. L., Hu, E. M., et al. 2004, AJ, 127, 180

Casertano, S., de Mello, D., Dickinson, M., et al. 2000, AJ, 120, 2747

Coe, D., Benítez, N., Sánchez, S. F., et al. 2006, AJ, 132, 926

Coleman, G. D., Wu, C.-C., \& Weedman, D. W. 1980, ApJS, 43, 393

Collister, A. A., \& Lahav, O. 2004, PASP, 116, 345
Cooper, M. C., Newman, J. A., Weiner, B. J., et al. 2008, MNRAS, 383, 1058 Coupon, J., Ilbert, O., Kilbinger, M., et al. 2009, A\&A, 500, 981 Dahlen, T., Mobasher, B., Faber, S. M., et al. 2013, ApJ, 775, 93

Davies, J. I., Baes, M., Bendo, G. J., et al. 2010, A\&A, 518, L48 Davies, J. I., Bianchi, S., Cortese, L., et al. 2012, MNRAS, 419, 3505 Davis, M., Faber, S. M., Newman, J. A., et al. 2003, Proc. SPIE, 4834, 161 Dawson, K. S., Schlegel, D. J., Ahn, C. P., et al. 2013, AJ, 145, 10 Dietrich, J. P., Erben, T., Lamer, G., et al. 2007, A\&A, 470, 821 Durrell, P. R., Côté, P., Peng, E. W., et al. 2014, ApJ, 794, 103 Eisenstein, D. J., Annis, J., Gunn, J. E., et al. 2001, AJ, 122, 2267

Erben, T., Hildebrandt, H., Lerchster, M., et al. 2009, A\&A, 493, 1197

Erben, T., Hildebrandt, H., Miller, L., et al. 2013, MNRAS, 433, 2545

Ferrarese, L., Côté, P., Cuillandre, J.-C., et al. 2012, ApJS, 200, 4 Fitzpatrick, E. L. 1986, AJ, 92, 1068

Gawiser, E., van Dokkum, P. G., Herrera, D., et al. 2006, ApJS, 162, 1

Gerdes, D. W., Sypniewski, A. J., McKay, T. A., et al. 2010, ApJ, 715, 823

Giovanelli, R., Haynes, M. P., Kent, B. R., et al. 2005, AJ, 130, 2598

Guzzo, L., Scodeggio, M., Garilli, B., et al. 2013, A\&A, 556, 108

Gwyn, S. D. J. 2012, AJ, 143, 38

Hanes, D. A., Côté, P., Bridges, T. J., et al. 2001, ApJ, 559, 812

Haynes, M. P., Giovanelli, R., Martin, A. M., et al. 2011, AJ, 142, 170

Heymans, C., Van Waerbeke, L., Miller, L., et al. 2012, MNRAS, 427, 146

Hildebrandt, H., Arnouts, S., Capak, P., et al. 2010, A\&A, 523, A31

Hildebrandt, H., Erben, T., Kuijken, K., et al. 2012, MNRAS, 421, 2355

Hildebrandt, H., Pielorz, J., Erben, T., et al. 2009a, A\&A, 498, 725

Hildebrandt, H., van Waerbeke, L., \& Erben, T. 2009b, A\&A, 507, 683

Ilbert, O., Arnouts, S., McCracken, H. J., et al. 2006, A\&A, 457, 841

Ilbert, O., Capak, P., Salvato, M., et al. 2009, ApJ, 690, 1236

Jouvel, S., Host, O., Lahav, O., et al. 2014, A\&A, 562, A86

Kent, B. R., Giovanelli, R., Haynes, M. P., et al. 2008, AJ, 136, 713

Kinney, A. L., Calzetti, D., Bohlin, R. C., et al. 1996, ApJ, 467, 38

Koo, D. C. 1985, AJ, 90, 418

Labbé, I., Franx, M., Rudnick, G., et al. 2003, AJ, 125, 1107

Laureijs, R., Amiaux, J., Arduini, S., et al. 2011, arXiv:1110.3193

Le Fèvre, O., Cassata, P., Cucciati, O., et al. 2013, A\&A, 559, A14

Le Fèvre, O., Vettolani, G., Garilli, B., et al. 2005, A\&A, 439, 845

Lilly, S. J., Le Fèvre, O., Renzini, A., et al. 2007, ApJS, 172, 70

Magnier, E. A., \& Cuillandre, J.-C. 2004, PASP, 116, 449

Markwardt, C. B. 2009, in ASP Conf. Ser. 411, Astronomical Data Analysis Software and Systems XVIII, ed. D. A. Bohlender, D. Durand, \& P. Dowler (San Francisco, CA: ASP), 251

McQuinn, M., \& White, M. 2013, MNRAS, 433, 2857

Muñoz, R. P., Puzia, T. H., Lançon, A., et al. 2014, ApJS, 210, 4

Newman, J. A. 2008, ApJ, 684, 88

Newman, J. A., Cooper, M. C., Davis, M., et al. 2013, ApJS, 208, 5

Prevot, M. L., Lequeux, J., Prevot, L., Maurice, E., \& Rocca-Volmerange, B. 1984, A\&A, 132, 389

Raichoor, A., \& Andreon, S. 2012, A\&A, 537, A88

Schlegel, D. J., Finkbeiner, D. P., \& Davis, M. 1998, ApJ, 500, 525

Scranton, R., Ménard, B., Richards, G. T., et al. 2005, ApJ, 633, 589

Skrutskie, M. F., Cutri, R. M., Stiening, R., et al. 2006, AJ, 131, 1163

Strader, J., Romanowsky, A. J., Brodie, J. P., et al. 2011, ApJS, 197, 33

Strauss, M. A., Weinberg, D. H., Lupton, R. H., et al. 2002, AJ, 124, 1810

York, D. G., Adelman, J., Anderson, J. E., Jr., et al. 2000, AJ, 120, 1579

Zhang, H., Peng, E. W., Côté, P., et al. 2014, ApJ, submitted

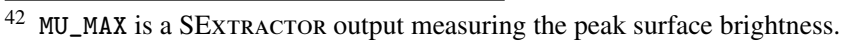

